JOURNAL OF ETHNOBIOLOGY

AND ETHNOMEDICINE

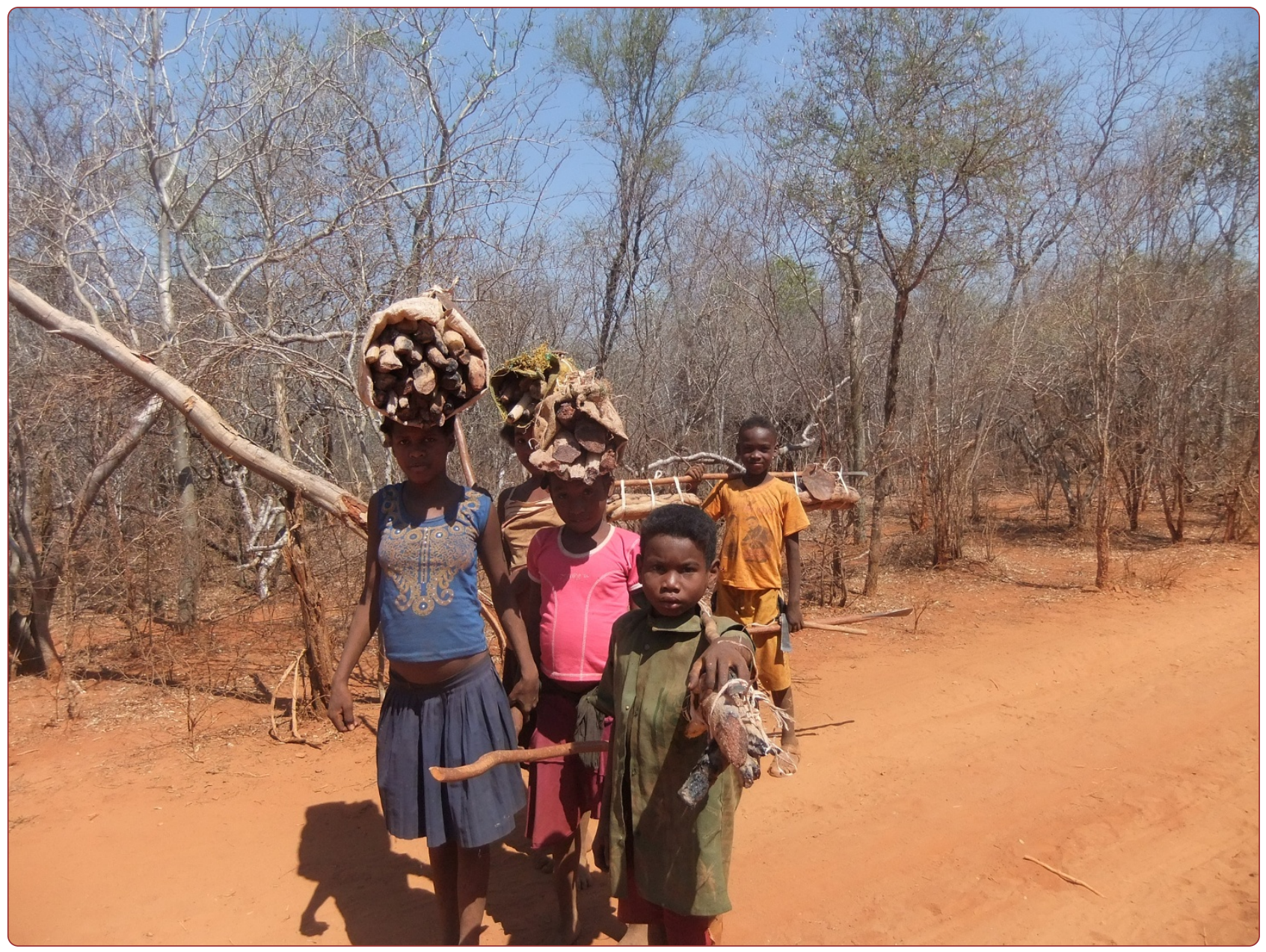

Effects of socio-economic household characteristics on traditional knowledge and usage of wild yams and medicinal plants in the Mahafaly region of south-western Madagascar

Andriamparany et al. 


\title{
Effects of socio-economic household characteristics on traditional knowledge and usage of wild yams and medicinal plants in the Mahafaly region of south-western Madagascar
}

\author{
Jessica N Andriamparany ${ }^{1}$, Katja Brinkmann ${ }^{1 *}$, Vololoniaina Jeannoda ${ }^{2}$ and Andreas Buerkert ${ }^{1}$
}

\begin{abstract}
Background: Rural households in the Mahafaly region of semi-arid SW-Madagascar strongly depend on the exploitation of natural resources for their basic needs and income regeneration. An overuse of such resources threatens the natural environment and people's livelihood. Our study focuses on the diversity and use of wild yams and medicinal plants.

Methods: We hypothesized that knowledge on the use of these resources highly depends on farmers' socio-economic household characteristics. To test this hypothesis, an ethnobotanical survey was conducted based on semi-structured interviews recording socio-economic base data and information on local knowledge of medicinal and wild yam species. This was followed by field inventories compiling plant material for botanical identification.

Results: Six species of wild yam and a total of 214 medicinal plants from 68 families and 163 genera were identified. Cluster and discriminant analysis yielded two groups of households with different wealth status characterized by differences in livestock numbers, off-farm activities, agricultural land and harvests. A generalized linear model highlighted that economic factors significantly affect the collection of wild yams, whereas the use of medicinal plants depends to a higher degree on socio-cultural factors.

Conclusions: Wild yams play an important role in local food security in the Mahafaly region, especially for poor farmers, and medicinal plants are a primary source of health care for the majority of local people. Our results indicate the influence of socio-economic household characteristics on the use of forest products and its intensity, which should be considered in future management plans for local and regional forest conservation.
\end{abstract}

Keywords: Discriminant analysis, Local knowledge, Medicinal plants, Socio-economic factors, Wild yams

\section{Background}

Madagascar constitutes one of the most important biodiversity hotspots worldwide with more than $90 \%$ of its plant and animal species being endemic, however, these resources are severely threatened by ecosystem degradation [1,2]. With a gross national income (GNI) per capita of \$828 [3], Madagascar ranks 151 out of 187 countries on the Human Development Index (HDI).

\footnotetext{
* Correspondence: tropcrops@uni-kassel.de

'Organic Plant Production and Agroecosystems Research in the Tropics and Subtropics, University of Kassel, Witzenhausen, Germany

Full list of author information is available at the end of the article
}

Altogether, $74 \%$ of the population lives in rural areas of which $78 \%$ are considered poor [4] and mostly depend on the direct exploitation of natural resources (fields, water, forests) for their livelihoods.

The arid south-western region of Madagascar, commonly referred to as the Mahafaly region, is the country's economically and climatically most disadvantaged area. It is characterised by high biotic endemism, listed as one of the 200 most important ecological regions in the world [5]. The subsistence production of the rural population comprises fishery, agriculture, livestock husbandry, and the collection of forest resources. Farmers' livelihoods and 
economic development is hampered by a low level of education, limited income alternatives and poor infrastructure. The productivity of the cropland is limited by highly unpredictable rainfall and soil fertility constraints very similar to those encountered in the West African Sahel $[6,7]$. Therefore, collection of forest products provides an important supplementary source of income [8], and an overuse of such resources threatens people's livelihood. Among these forest products, the collection of wild yam (Dioscorea spp.) species and medicinal plants were identified as important for the local population [8,9], as they contribute to the well-being of rural households in terms of direct use, human nutrition and income generation.

Medicinal plants constitute an important alternative to conventional medicine, especially for poor communities in rural areas without access to health services and they display a very large diversity in terms of species number [10]. According to the World Health Organization, approximately $80 \%$ of the world's inhabitants rely predominantly on traditional medicine for their primary health care [11]. Of approximately 13,000 species present in Madagascar, about 3,500 are reported to have medicinal properties [12]. Madagascar has also a rich diversity of yam with altogether 40 species of which 27 are endemic and most of them have edible tubers [13], which are a staple food in many tropical countries. Wild yams have been reported to play an important role in rural household livelihoods system where they are traditionally eaten during periods of food insecurity [14]. The genus Dioscorea is distributed in various areas in Madagascar, but 24 species including 20 endemics were observed in the south western region [15]. These species are all edible, but the intensity of local usage depends on taste, local needs, market prices, location and harvested amounts. Other factors governing tuber use are differences in culture, gender, language, ethnicity, political belief system, personal preferences, appropriation skills and the availability of these resources in collection areas [16].

Detailed information on the importance of wild yams and medicinal plants for people's livelihood and the factors influencing the intensity of their use are urgently required for natural resource management policy and planning and is lacking for SW-Madagascar. Therefore, the objective of this study was to analyse the diversity and use of wild yams and medicinal plants in the Mahafaly region, and to identify their role in the livelihoods of local people. We hypothesized that local knowledge on the usage of wild yams and medicinal plants depends on the socio-economic conditions and wealth status of households. Thereby, poorer households depend to a higher degree on forest resources and have a higher knowledge on their use than well-off farmers.

\section{Materials and methods}

\section{Description of the study area}

The study area is situated in the northern part of the Mahafaly region. The studied villages are located on the adjacent coast (littoral) and on the west side (plateau) of the Tsimanampetsotsa National Park $\left(24^{\circ} 03^{\prime}-24^{\circ} 12^{\prime} \mathrm{S}\right.$, $43^{\circ} 46^{\prime}-43^{\circ} 50^{\prime} \mathrm{E}$; Figure 1$)$. The area is characterized by a dry and spiny forest vegetation with the highest level of endemism in plant species registered in Madagascar (48\% of genera and $95 \%$ of species; [17]). The natural vegetation consists of a deciduous forest characterized by drought tolerant woody species of Didieraceae and Euphorbiaceae, xerophytic bushland and savannah. In the littoral zone dry forests on sandy soil dominate while on the plateau dry and spiny forests on tertiary limestone or ferruginous soil occur [18]. The semi-arid climate is characterized by an annual mean temperature of $24^{\circ} \mathrm{C}$ and a highly variable annual rainfall ranging between $300-350 \mathrm{~mm}$ in the littoral and $400-450 \mathrm{~mm}$ on the plateau [19]. The dry season lasts nine to ten months and the rainy season five months from November to April. The unreliability and unpredictability of rainfall is one of the major factors limiting agricultural production by the predominantly small holder farmers and herders, which partly rely on forest products to fulfil their daily needs throughout the year. During the past 40 years forest cover declined by $45 \%$ due to slash and burn agriculture and uncontrolled bushfires [20,21]. In addition, the region has the lowest education rate of Madagascar and the majority of the households were classified as poor [22] in combination with a lack of basic health services and infrastructure. Altogether, $41 \%$ of the local population on the Mahafaly region is affected by food insecurity and famine [23]. Rapid population growth and the recent expansion of the Tsimanampetsotsa National Park (from 42,200 to 203,000 ha in 2007) have increased the pressure on the forests resources in and outside the park area $[21,24,25]$. Combined with the effects of climate change this leads to an increasing over-use of the natural resources in the Mahafaly region.

In the Mahafaly region wild yams are used to supplement cassava (Manihot esculanta Krantz) and maize (Zea mays L.), especially during hunger periods ('Kere'). Local reports indicate that during the past years the amount of harvested wild yam tubers has strongly increased given a rising insufficiency of crop production.

\section{Field survey}

The field work was conducted from June to December 2012 in five villages that were part of a larger village and household survey [21,26]: (1) Efoetse in the littoral (S 244' 42,41" - E 4341'54,78'), (2) Ampotake (S 2352'

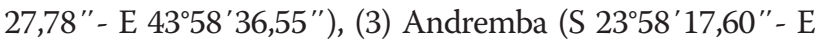




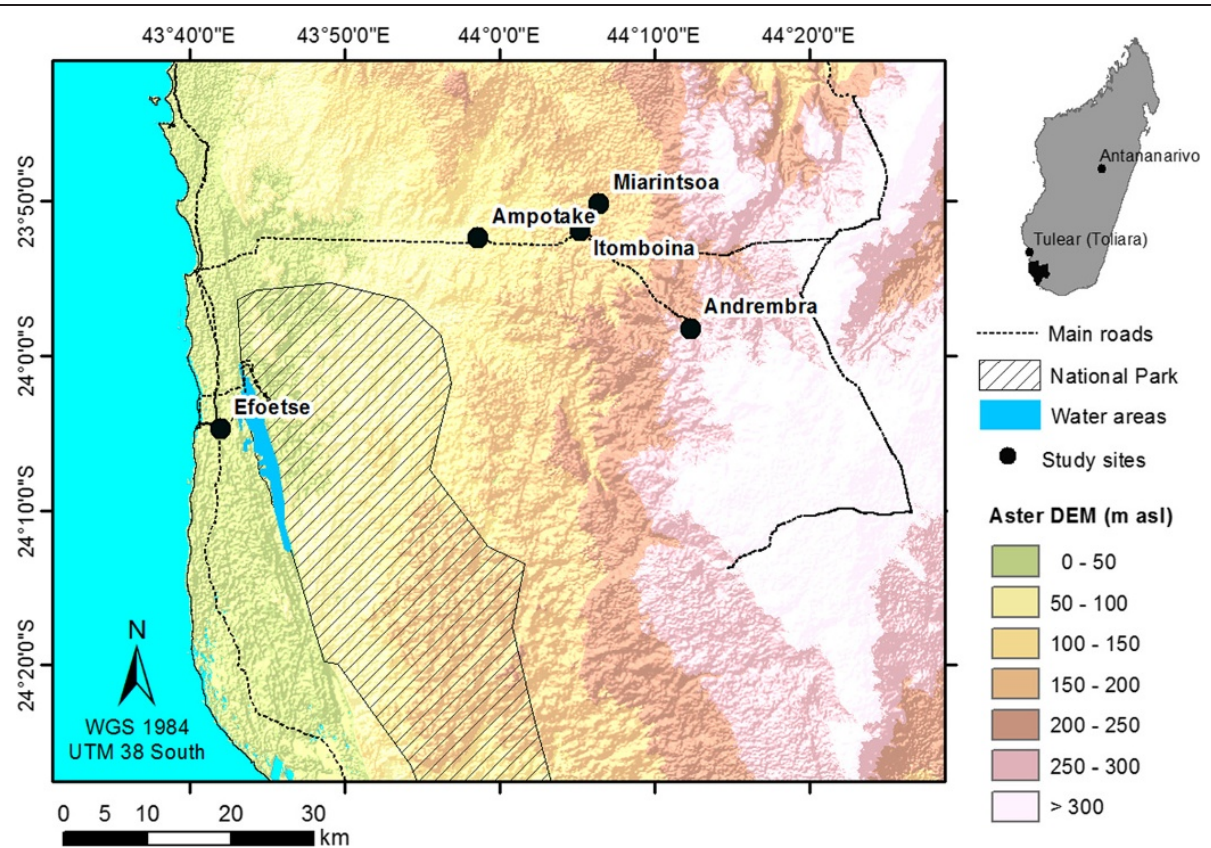

Figure 1 Location of the study area in the Mahafaly region of SW-Madagascar.

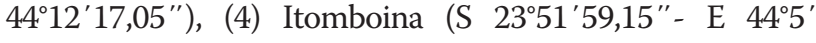

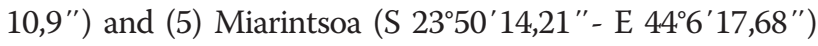
on the plateau. Village selection was based on (1) market accessibility, (2) distance to the national park, (3) intensity of forest product collection of village inhabitants and (4) diversity of household activities. For each village, 50 households $(\mathrm{HH})$ were randomly selected based on a complete household list (total $\mathrm{N}=250$ ). Pre-testing interviews and field observations were performed with key informants selected by snowball sampling [27]. Semi-structured interviews [28] were conducted with the household head after we received his consent. The Code of Ethics of the International Society of Ethnobiology was followed. If household head disagreed to take part in an interview, an alternative household was chosen based on an existing household list of the village. The questionnaire was divided in three thematic sections: (1) Information on socio-cultural and economic characteristic (family size, source of income, agricultural harvest, origin of the head and spouse, land area available for cultivation, livestock owned, harvest satisfaction, education level, ethnic group, religion, gender affiliation and age of respondents); (2) Household consumption, collection and use of wild yam species; (3) Medicinal plants and the knowledge about their uses. Respondents were also asked about specific plant parts used and the habitat from which they collected the plant material. All interviews were supplemented with field observations and forest walks. Since informants were only able to mention the local species name, plant specimen were collected in the field to establish a digital herbarium of inventoried specimens for botanical identification [29] in the Herbarium of the Botanical and Zoological Parc of Tsimbazaza (PBZT) in Antananarivo (Madagascar), following the nomenclature of the Tropicos database of the Missouri Botanical Gardens [30].

In the absence of any formal ethics committee the concept, content and questions related to this study conducted within the participatory SuLaMa (sustainable Land Management in South-Western Madagascar) project (www.sulama.de) were discussed and approved at the governmental and the village level in several meetings as were the outcomes of the interviews.

\section{Data analysis}

The consumption, collection intensity and usage of wild yams were analysed using the following interview data: number of species collected, frequency of collection per month, period of collection per year, average number of tubers collected per collection event (estimated by the number of harvest holes), number of collectors per households, type of consumption (staple or additional food) and sale of tubers. The types of medicinal usage were categorized in different medicinal categories according to Cook [31]. To estimate the informant knowledge on the use of medicinal plants, the diversity of medicinal plant uses [32,33] was calculated for each informant. The species $\left(U_{\mathrm{S}}\right)$ and the family use values (FUV) were computed (Table 1) [34,35] to compare the importance of plant species and families. 
Table 1 Ethnobotanical indices used for measuring informant's medicinal plant knowledge in the Mahafaly region of SW-Madagascar

\begin{tabular}{|c|c|c|}
\hline Indices & Calculation & Description \\
\hline Diversity of medicinal plant use (D) & $\begin{array}{l}\mathrm{D}=1 / \sum \mathrm{Pi}^{2} \text {, where } \mathrm{Pi}^{2} \text { is equal to the number } \\
\text { of times a species was mentioned by informant } \\
\text { ' } \mathrm{i} \text { ' divided by the total number of informants } \\
\text { answer. }\end{array}$ & $\begin{array}{l}\text { Simpson's Reciprocal Index [32], adapted by } \\
\text { [33]. Measures how many medicinal plant } \\
\text { species an informant uses and how evenly } \\
\text { his uses are distributed among the species. }\end{array}$ \\
\hline Species use value $\left(U V_{S}\right)$ & $\begin{array}{l}U V_{S}=\sum U V_{i s} / n_{i} \text {, where } U V_{i s} \text { is the sum of the } \\
\text { total number of use citations by all informants } \\
\text { for a given species and ni is the total number } \\
\text { of informants. }\end{array}$ & $\begin{array}{l}\text { Evaluates the relative importance of each } \\
\text { plant species based on its relative use among } \\
\text { informants [34], adapted by [35]. }\end{array}$ \\
\hline Family use value (FUV) & $\begin{array}{l}\text { FUV }=\sum U V_{s} / n_{s} \text {, where } \sum U V_{S} \text { is the sum of } \\
\text { species use value (UVs) within a family and } \\
n_{s} \text { the number of species within a family. }\end{array}$ & $\begin{array}{l}\text { Evaluates the use importance of a given plant } \\
\text { family [34]. }\end{array}$ \\
\hline
\end{tabular}

All statistical analyses were carried out using SPSS 17.0. A two-step cluster analysis was used to identify household groups based on socio-economic characteristics and plant use patterns. The existence of collinearity was tested based on correlation coefficients and suspicious data was removed from the dataset resulting in the following parameters used for cluster analysis: Education level, agricultural harvest, household activities, family size, tropical livestock units, agricultural area, medicinal plants used, number of medicinal uses and diversity of medicinal plant use (D), wild yam species collected, amount of tubers harvested (number of holes harvested for each collection), frequency of collection, sale, collection period and use of wild yams.

To evaluate the contribution of each variable in separating the resulting households groups, a Discriminant Analysis (DA) was conducted using the standardized canonical coefficients, canonical correlation coefficients, Eigen value and Wilk's Lambda. A structure coefficient matrix was established which allowed to assess the importance of each variable in relation to the discriminant function.

A One Way ANOVA (Analysis of variance) was performed to compare the differences of knowledge and use between communities in relation to their location (villages). Additionally, we used Jaccard's similarity index, which was based on species usage data to determine the similarity of species usage among villages [36].

To determine which cultural and socio-economic variables influence the use intensity and knowledge on medicinal plants and wild yams (response variables), we used a Generalized Linear Model (GLM) based on a Poisson distribution. The GLM consisted of two models with eight response variables, which explain the relationship between predictors and the knowledge on medicinal plants (number of medicinal plants used) and the use of wild yams (frequency of yam collection per month). The performance and the fit of the models were assessed using the Akaike Information Criterion (AIC; [37]). In each model, we only included main effects and choose the Type III analyses and Wald chi-square as statistical tests. The 0.05 significance level was used to assess if an independent variable related significantly to a dependent variable.

\section{Results and discussions}

\section{Socio-economic characteristics of the interviewed} households

Average household size varied between 6.3 persons in Itomboina and 7.2 persons in Miarintsoa (Table 2) whereby big households typically comprised a polygamous household head. Thus, each sub-family might live separately, but all family members eat together and share the same income. The education level of the households was highly variable across the villages, but in general, $30 \%$ of interviewed households did not receive formal education and only half visited at least the first year of primary school. The village with the highest rate of illiteracy, Ampotake, had no school. However, in Efoetse, where public and even private schools are available, literacy was high. The majority of the households comprise small holder farmers, which conduct different off-farm activities for cash income generation, such as salaried work, artisanal activities, trading, fishing, charcoal production or the collection of wood and other forest resources. The average household's agricultural area was 2.2 ha of which some was partly left uncultivated due to heavy weed encroachment or a perceived decline in soil productivity. For the majority of households, periods of food insecurity due to unpredictable and insufficient rainfall are frequent and people heavily depend on supplementary off-farm income. Most of the household heads were born in the village where they live, only $26 \%$ are immigrants. The majority of households (60\%) has traditional religious beliefs (ancestor reverence) and conduct ritual practices, while 30\% are Christian (Catholic, Protestant or Anglicans). 
Table 2 Socioeconomic characteristics of the interviewed households (HH) in the five villages of the Mahafaly region in SW-Madagascar

\begin{tabular}{|c|c|c|c|c|c|c|c|}
\hline \multicolumn{2}{|l|}{ Characteristics } & $\begin{array}{l}\text { Ampotaka } \\
(\mathrm{n}=55)\end{array}$ & $\begin{array}{l}\text { Andremba } \\
(\mathrm{n}=50)\end{array}$ & $\begin{array}{l}\text { Itomboina } \\
(\mathrm{n}=50)\end{array}$ & $\begin{array}{l}\text { Miarintsoa } \\
(\mathrm{n}=50)\end{array}$ & $\begin{array}{l}\text { Efoetse } \\
(n=50)\end{array}$ & Total \\
\hline \multicolumn{2}{|l|}{ Age of the respondents } & $41.7 \pm 17.3$ & $44.2 \pm 15.5$ & $46.7 \pm 18.3$ & $40.4 \pm 17.6$ & $42.6 \pm 19.9$ & $43.1 \pm 17.8$ \\
\hline \multicolumn{2}{|l|}{ Family size } & $6.8 \pm 3.9$ & $6.4 \pm 3$ & $6.3 \pm 3.3$ & $7.2 \pm 3.7$ & $6.7 \pm 2.3$ & $6.7 \pm 3.3$ \\
\hline \multicolumn{2}{|l|}{ TLU } & $1.6 \pm 3.1$ & $5.1 \pm 9.2$ & $4.8 \pm 7.5$ & $6.9 \pm 10.9$ & $9.2 \pm 12.8$ & $5.5 \pm 9.5$ \\
\hline \multicolumn{2}{|l|}{ Land owned (ha) } & $1.6 \pm 1.4$ & $1.7 \pm 1.1$ & $2.3 \pm 2.1$ & $2.7 \pm 2.1$ & $2.7 \pm 2.1$ & $2.2 \pm 1.8$ \\
\hline \multirow[t]{3}{*}{ Agricultural harvest (\%) } & Low & 44 & 36 & 62 & 32 & 14 & 38.0 \\
\hline & Medium & 50 & 42 & 36 & 52 & 66 & 49.2 \\
\hline & High & 6 & 20 & 2 & 16 & 20 & 12.8 \\
\hline \multirow[t]{3}{*}{ HH activities (\%) } & Low & 42 & 38 & 38 & 24 & 46 & 37.6 \\
\hline & Medium & 36 & 46 & 44 & 46 & 40 & 42.4 \\
\hline & High & 22 & 16 & 18 & 30 & 14 & 20.0 \\
\hline \multirow[t]{3}{*}{ Education level } & Low & 52 & 22 & 32 & 16 & 24 & 29.2 \\
\hline & Visit primary school & 34 & 56 & 50 & 54 & 54 & 49.6 \\
\hline & Finish primary school & 14 & 22 & 18 & 30 & 22 & 21.2 \\
\hline \multirow[t]{2}{*}{ Origin of the head of the $\mathrm{HH}(\%)$} & Born in the village & 28 & 10 & 40 & 38 & 18 & 26.8 \\
\hline & Not born in the village & 72 & 90 & 60 & 62 & 82 & 73.2 \\
\hline \multirow[t]{2}{*}{ Gender of the respondent (\%) } & Male & 60 & 70 & 64 & 74 & 84 & 70.4 \\
\hline & Female & 40 & 30 & 36 & 26 & 16 & 29.6 \\
\hline \multirow[t]{3}{*}{ Religion (\%) } & No religion & 14 & 8 & 4 & 6 & 17 & 9.7 \\
\hline & Traditional & 60 & 62 & 64 & 58 & 55.3 & 59.9 \\
\hline & Christian & 26 & 30 & 32 & 36 & 27.7 & 30.4 \\
\hline
\end{tabular}

\section{Diversity and traditional use of plants Wild yams}

Altogether, six endemic species of wild yam were identified as potential food resource in the Mahafaly region: Dioscorea ovinala Baker (local name: 'Angily'), Dioscorea alatipes Burk. \& H. Perr. ('Ovy'), Dioscorea nako H. Perr. ('Fandra'), Dioscorea fandra H. Perr. ('Andraha'), Dioscorea bemandry Jum. \& H. Perr. ('Baboky') and Dioscorea soso Jum. \& H. Perr. ('Sosa'). Two thirds of the interviewed households (70\%) were collecting wild yams. Yam collection was only uncommon in Efoetse where yams could be purchased from nearby markets. This is mainly due to the limited access to forest and yam resources in the littoral zone, where larger forest areas are lacking except of the Tsimanampetsotsa National Park area. In addition, wild yam species are relatively rare on the adjacent side of the national park where only $D$. nako occurs

Wild yam tubers are used as a staple food by $42 \%$ of the households where they substitute cassava, maize or sweet potato (Ipomoea batatas L.), especially in villages situated near forest areas, where daily plant collection is possible. Respondents mentioned that they eat yams before the meal to reduce the quantity of staple food during the lean season. D. alatipes was most frequently collected (99\% of yams collecting households), mainly because of its sweet taste and nutritional value. The so called water yam, D. bemandry, was also important and collected by $88 \%$ of households, because of its sweet taste and its big and long tubers (50-120 cm long). D. soso had the lowest collection rate (34\% of households) given its scarce occurrence in the surrounding forests, although its taste is also appreciated by the local population.

\section{Medicinal plants}

Altogether, 221 medicinal plants are used by the local people in the Mahafaly region (Table 3) of which 214 plant species were taxonomically identified and belong to 163 genera in 68 plant families. These plants are used to treat 46 diseases of human and livestock. Most species belonged to the Fabaceae (34 species), followed by Apocynaceae (17 species), Euphorbiaceae (16 species) and Malvaceae (10 species; Figure 2). Some families, such as the Aizoaceae, Aristolochiaceae, Flacourtiaceae, Myrtaceae, Sapotaceae, and Moringaceae were represented by only one species. Plant families with the highest FUV are Rutaceae (1.53), Capparaceae (1.37), Hernandiaceae (1.27) and Asteraceae (1.24). Among the 46 uses reported, the most common are digestive 
Table 3 List of medicinal plants species used in the Mahafaly region, SW-Madagascar

\begin{tabular}{|c|c|c|c|c|c|c|c|}
\hline Scientific name & Family & Local name & Use value & Citation (\%) & Habitat & Parts used & Voucher number* \\
\hline Cedrelopsis grevei Baill. & Rutaceae & Katrafay & 3.06 & 99.6 & Forest & $\mathrm{Lv}, \mathrm{Br}, \mathrm{Tr}$ & R. Rabevohitra 2390 \\
\hline Croton sp. 6 & Euphorbiaceae & Tambio & 3 & 0.4 & Forest & $\mathrm{Sb}$ & - \\
\hline Boscia tenuifolia A. Chev. & Capparaceae & Lalangy & 2 & 0.4 & Forest & $\mathrm{Ar}$ & - \\
\hline Pluchea grevei (Baill.) Humbert & Asteraceae & Samonty & 1.91 & 5.5 & Forest & LV & J.Bosser 9917 \\
\hline Aloe divaricata A. Berger & Xanthorrhoeaceae & Vahondrandro & 1.87 & 100 & Forest & $L x$ & Reynold 7860 \\
\hline Cadaba virgata Bojer & Capparaceae & Tsihariharinaliotse & 1.5 & 0.9 & Forest & $\operatorname{Ar}$ & Bewerley Lewis 534 \\
\hline Tamarindus indica $\mathrm{L}$. & Fabaceae & Kily & 1.47 & 59.2 & Forest, Fallow & $\mathrm{Lv}, \mathrm{Br}, \mathrm{Fr}$ & Thomas B. Croat 31108 \\
\hline Neobeguea mahafaliensis Leroy, Jean F. P. & Meliaceae & Handy & 1.44 & 91.1 & Forest & $\mathrm{Sb}, \mathrm{Tr}$ & R. Decary 16206 \\
\hline Croton sp. 4 & Euphorbiaceae & Zalazala & 1.38 & 14.5 & Forest & $\mathrm{Br}$ & - \\
\hline Ficus lutea Vahl. & Moraceae & Amonta & 1.38 & 6.8 & Forest & $\mathrm{Ar}$ & G McPherson 14634 \\
\hline Psiadia angustifolia (Humbert) Humbert & Asteraceae & Ringandringa & 1.38 & 22.1 & Forest & LV & RN 3806 \\
\hline Sida rhombifolia L. & Malvaceae & Mandravasarotse & 1.38 & 6.8 & Fallow & $\operatorname{Ar}$ & Thomas B. Descoings 30725 \\
\hline Croton geayi Leandri & Euphorbiaceae & Pisopiso & 1.36 & 72.3 & Forest & $\mathrm{Sb}, \mathrm{Br}$ & H. Humbert 2397 \\
\hline Lemuropisum edule $\mathrm{H}$. Perrier & Fabaceae & Berotse & 1.36 & 10.6 & Forest & $\mathrm{Sb}$ & J. Bosser 1984 \\
\hline Acacia sakalava Drake & Fabaceae & Roymena & 1.33 & 1.3 & Savanna, Forest & $\mathrm{Ar}$ & J.F. Villiers 4056 \\
\hline Dalbergia sp. & Fabaceae & Manary & 1.33 & 12.8 & Forest & $\mathrm{Br}$ & - \\
\hline Acacia bellula Drake & Fabaceae & Rohy & 1.3 & 14 & Forest & $\operatorname{Ar}$ & R. Ranaivojaona 492 \\
\hline Hernandia voyronii Jum. & Hernandiaceae & Hazomalany & 1.3 & 4.3 & Forest & $\operatorname{Tr}$ & J.Bosser 9178 \\
\hline Euphorbia tirucalli L. & Euphorbiaceae & Laro & 1.29 & 53.6 & Forest & $\mathrm{Lv}, \mathrm{St}$ & P.B. Phillipson 2480 \\
\hline Coffea grevei Drake ex A.Chev & Rubiaceae & Hazombalala & 1.28 & 31.5 & Forest & $\mathrm{Sb}, \mathrm{Ar}$ & C.C.H. Jonngkind 3746 \\
\hline Aloe vaombe Decorse \& Poisson & Xanthorrhoeaceae & Vahombe & 1.25 & 37.9 & Forest & $L x$ & H. Humbert 5418 \\
\hline Cynanchum mahafalense Jum. \& H. Perrier & Apocynaceae & Vahimasy & 1.25 & 19.2 & Forest & $\mathrm{Sb}, \mathrm{St}$ & B. Descoings 3251 \\
\hline Citrullus lanatus (Thunb.) Mansf. \& Naka & Cucurbitaceae & Voamanga & 1.24 & 20.9 & Crop field & $\operatorname{Ar}$ & J. Bosser 13567 \\
\hline Croton kimosorum Leandri & Euphorbiaceae & Zanompoly & 1.24 & 26.8 & Forest & $\mathrm{Br}$ & J. Bosser 10429 \\
\hline Gyrocarpus americanus Jacq. & Hernandiaceae & Kapaipoty & 1.24 & 10.6 & Forest & LV & P.B. Phillipson 2350 \\
\hline Operculicarya decaryi H. Perrier & Anacardiaceae & Jabihy & 1.24 & 52.3 & Forest & $\mathrm{Br}, \mathrm{Tr}$ & P. Morat 696 \\
\hline Tetrapterocarpon geayi Humbert & Fabaceae & Hazolava/Noaovy & 1.24 & 38.7 & Forest & $\mathrm{Sb}, \mathrm{Br}$ & B. Descoings 1433 \\
\hline Erythroxylum retusum Baill. ex O.E. Schulz & Erythroxylaceae & Montso & 1.23 & 71.9 & Forest & LV & P.B. Phillipson 2464 \\
\hline Mangifera indica $\mathrm{L}$. & Anacardiaceae & Mangavato & 1.23 & 4.7 & Crop field & $\mathrm{Br}$ & _- \\
\hline Polycline proteiformis Humbert & Asteraceae & Zira & 1.22 & 3.4 & Forest & Sb,ar & J. Bosser 248 \\
\hline Leptadenia madagascariensis Decne. & Apocynaceae & Taritarika/Mozy & 1.21 & 46.4 & Forest & $\mathrm{Sb}, \mathrm{Ar}$ & B. Descoings 1243 \\
\hline Ruellia anaticollis Benoist & Acanthaceae & Reforefo & 1.21 & 7.2 & Forest & $\operatorname{Ar}$ & P.B.Phillipson 1795 \\
\hline Bulbostylis xerophila H. Cherm. & Cyperaceae & Foentany & 1.2 & 2.1 & Forest & $\mathrm{Ar}$ & M.R. Decary 8531 \\
\hline
\end{tabular}


Table 3 List of medicinal plants species used in the Mahafaly region, SW-Madagascar (Continued)

\begin{tabular}{|c|c|c|c|c|c|c|c|}
\hline Grewia sp. & Malvaceae & Malimatse & 1.2 & 2.1 & Forest & $\mathrm{Br}$ & - \\
\hline Mundulea sp. 1 & Fabaceae & Sofasofa & 1.2 & 6.4 & Forest & $\operatorname{Ar}$ & - \\
\hline Oeceoclades decaryana (H. Perrier) Garay \& P. Taylor & Orchidaceae & Hatompototse & 1.2 & 2.1 & Forest & St & Gordon Mc Pherson 17376 \\
\hline Paederia grandidieri Drake & Rubiaceae & Tamboro & 1.19 & 11.1 & Forest & LV & P.B. Phillipson 2810 \\
\hline Salvadora angustifolia Turill & Salvadoraceae & Sasavy & 1.19 & 79.6 & Forest & $\mathrm{Lv}, \mathrm{Sb}$ & P.B. Phillipson 3711 \\
\hline Vanilla madagascariensis Rolfe & Orchidaceae & Amalo & 1.19 & 8.1 & Forest & St & - \\
\hline Aristolochia acuminate Lamk. & Aristolochiaceae & Totonga & 1.18 & 41.3 & Forest & $\mathrm{Sb}$ & P. Morat 3512 \\
\hline Commiphora lamii H. Perrier & Burseraceae & Holidaro & 1.17 & 5.1 & Forest & $\mathrm{Br}$ & C.C.H. Jongkind 3681 \\
\hline Cassia siamea Lam. & Fabaceae & Farefare & 1.16 & 21.3 & Forest & $\mathrm{Br}$ & M. B. Dupuy M98 \\
\hline Didierea madagascariensis Baill. & Didieraceae & Sono & 1.16 & 12.8 & Forest & $\operatorname{Tr}$ & D. Lorence 1928 \\
\hline Securinega perrieri Leandri & Phyllanthaceae & Hazomena & 1.16 & 10.6 & Forest & LV & Herb., Inst.Sci. Mad. 4497 \\
\hline Commiphora mahafaliensis Capuron & Burseraceae & Maroampotony & 1.15 & 8.5 & Forest & $\operatorname{Ar}$ & - \\
\hline Cynanchum grandidieri Liede \& Meve & Apocynaceae & Betondro & 1.15 & 24.7 & Forest & $\mathrm{Sb}$ & - \\
\hline Indigofera compressa Lam. & Fabaceae & Hazomby & 1.15 & 36.6 & Forest & $\operatorname{Ar}$ & M.R. Decary 9147 \\
\hline Ipomoea pes-caprae (L.) R. Br. & Convolvulaceae & Fobo & 1.15 & 8.5 & Seaside & $\mathrm{Sb}$ & Robert W. Books 19 \\
\hline Solanum hippophaenoïdes Bitt. & Solanaceae & Hazonosy & 1.15 & 25.5 & Forest & $\mathrm{Lv}, \mathrm{Sb}$ & - \\
\hline Croton sp. 5 & Euphorbiaceae & Andriambolafotsy & 1.14 & 3 & Forest & Lv & - \\
\hline Mundulea sp. 2 & Fabaceae & Taivosotse & 1.14 & 3 & Forest & $\mathrm{Ar}$ & - \\
\hline Zygophyllum depauperatum Drake & Zygophyllaceae & Filatatao & 1.14 & 3 & Forest & Lv & J. Bosser 10129 \\
\hline Blepharis calcitrapa Benoist & Acanthaceae & Sitsitse & 1.13 & 19.6 & Forest & $\mathrm{Sb}$ & H. Humbert 5136 \\
\hline Commiphora monstruosa (H. Perrier) Capuron & Burseraceae & Taraby & 1.13 & 19.2 & Forest & $\mathrm{Ar}, \mathrm{Tr}$ & - \\
\hline Cynanchum perrieri Choux & Apocynaceae & Ranga & 1.13 & 66.8 & Forest & St & Labat J-N 2414 \\
\hline Henonia scoparia Moq. & Amaranthaceae & Fofotse & 1.13 & 10.2 & Forest & LV & M.R. Decary 2531 \\
\hline Hypoestes phyllostachya Baker & Acanthaceae & Fotivovona & 1.13 & 13.6 & Forest & $\operatorname{Ar}$ & J. Bosser 43 \\
\hline Indigofera mouroundavensis Baill. & Fabaceae & Sambobohitse & 1.13 & 3.4 & Forest & $\mathrm{Sb}$ & Jacqueline \& M. Peltier 3171 \\
\hline Opuntia sp. 2 & Cactaceae & Raketamena & 1.13 & 6.4 & Crop field, Fallow & $\mathrm{Sb}$ & - \\
\hline Stereospermum nematocarpum DC. & Bignoniaceae & Mahafangalitse & 1.13 & 23.4 & Forest & $\mathrm{Br}$ & Herb. Inst. Sci. Mad. 4630 \\
\hline Streblus sp. & Moraceae & Hazondranaty & 1.13 & 20.4 & Forest & Sb.Tr & \\
\hline Zea mays $L$. & Poaceae & Tsako & 1.13 & 6.4 & Crop field & $\mathrm{Fr}$ & - \\
\hline Ziziphus spina-christi (L.) Willd. & Rhamnaceae & Tsinefo & 1.13 & 34.5 & Crop field, Fallow & $\mathrm{Br}$ & J. Bosser 416 \\
\hline Euphorbia stenoclada Baill. & Euphorbiaceae & Samata & 1.12 & 28.9 & Forest & $\mathrm{Lv}, \mathrm{Sb}$ & RN 4768 \\
\hline Grewia leucophylla Capuron & Malvaceae & Fotilambo & 1.12 & 7.2 & Forest & $\mathrm{Sb}, \mathrm{Br}$ & Michelle Sauther 23 \\
\hline Rhigozum madagascariense Drake & Bignoniaceae & Hazonta & 1.12 & 17.9 & Forest & $\operatorname{Ar}$ & J. Bosser 14420 \\
\hline
\end{tabular}


Table 3 List of medicinal plants species used in the Mahafaly region, SW-Madagascar (Continued)

\begin{tabular}{|c|c|c|c|c|c|c|c|}
\hline Grewia humblotii Baill. & Malvaceae & Sely & 1.11 & 26.4 & Forest & $\mathrm{Sb}, \mathrm{Br}$ & - \\
\hline Lasiocladus anthospermifolius Bojer ex Nees & Acanthaceae & Maintemaso & 1.11 & 24.3 & Forest & $\mathrm{Lv}, \mathrm{Sb}$ & J.N. Labat 2696 \\
\hline Cajanus cajan (L.) Millsp. & Fabaceae & Ambatry & 1.1 & 15.3 & Crop field & $\operatorname{Ar}$ & Thomas B. Croat 32106 \\
\hline Cynanchum nodosu (Jum. \& H. Perrier) Desc. & Apocynaceae & Try & 1.1 & 24.3 & Forest & $\mathrm{Sb}$ & P.B. Phillipson 1671 \\
\hline Adenia olaboensis Claverie & Passifloraceae & Hola & 1.09 & 4.7 & Forest & $L x$ & Jacqueline \& M. Peltier 1396 \\
\hline Azima tetracantha Lam. & Salvadoraceae & Tsingilo & 1.09 & 9.4 & Forest & LV & M.R Decary 3470 \\
\hline Hydnora esculenta Jum. \& H. Perrier & Hydnoraceae & Voantany & 1.09 & 9.8 & Forest & $\mathrm{Sb}$ & Herb., Inst.sci. Mad. 2 \\
\hline Sclerocarya birrea subsp. caffra (Sond.) Kokwaro & Anacardiaceae & Sakoa/Sakoamanga & 1.09 & 38.7 & Savana & $\mathrm{Lv}, \mathrm{Br}$ & D.J. Mabberley 732 \\
\hline Secamone tenuifolia Decne. & Apocynaceae & Langolora & 1.09 & 14.5 & Forest & $\mathrm{Sb}$ & J. Bosser 17209 \\
\hline Abutilon indicum (L.)Sweet & Malvaceae & Lahiriky & 1.08 & 22.1 & Forest, Fallow & $\operatorname{Ar}$ & L.J. Dorr 4056 \\
\hline Capuronianthus mahafalensis J.-F. Leroy & Meliaceae & Ringitse & 1.08 & 5.1 & Forest & $\mathrm{Sb}$ & - \\
\hline Mollugo decandra Scott-Elliot & Molluginaceae & Andriamanindry & 1.08 & 10.2 & Forest & $\operatorname{Ar}$ & H. Humbert 5293 \\
\hline Moringa drouhardii Jum. & Moringaceae & Maroserana & 1.08 & 5.5 & Forest & $\mathrm{Ar}$ & B. Descoings 2411 \\
\hline $\begin{array}{l}\text { Pentarhopalopilia madagascariensis Cavaco } \\
\text { \& Keraudren }\end{array}$ & Opiliaceae & Fandriandambo & 1.08 & 10.2 & Forest & $\mathrm{Ar}$ & B. Descoings 1214 \\
\hline Ximenia perrieri Cavaco \& Keraudren & Ximeniaceae & Kotro & 1.08 & 26.8 & Forest & $\mathrm{Lv}, \mathrm{Sb}$ & Rauh 1221 \\
\hline $\begin{array}{l}\text { Cymbopogon excavatus (Hochst.) Stapf ex Burtt } \\
\text { Davy }\end{array}$ & Poaceae & Ahibero & 1.07 & 1.7 & Forest & Lv & Bosser 5208 \\
\hline Avicennia marina (Forssk.) Vierh. & Acanthaceae & Afiafy & 1.06 & 3.8 & Forest & $\mathrm{Br}$ & James L. Zarucchi 7552 \\
\hline Enterospermum pruinosum (Baill.) Dubard \& Dop & Rubiaceae & Mantsake & 1.06 & 7.2 & Forest & $\mathrm{Br}$ & - \\
\hline Hyphaene sp. & Arecaceae & Satra & 1.06 & 22.1 & Crop field & $\mathrm{Lv}, \mathrm{Sb}$ & \\
\hline Zingiber officinale Roscoe & Zingiberaceae & Sakaviro & 1.06 & 14.5 & Crop field & $\mathrm{Sb}$ & M.R. Decary 1440 \\
\hline Chloroxylon falcatum Capuron & Rutaceae & Mandakolahy & 1.05 & 35.3 & Forest & St & - \\
\hline Jatropha mahafalensis Jum. \& H.Perrier & Euphorbiaceae & katratra & 1.05 & 46 & Forest & $L v, L x$ & H. Humbert 2521 \\
\hline $\begin{array}{l}\text { Pentatropis nivalis subsp. madagascariensis (Decne.) } \\
\text { Liede \& Meve }\end{array}$ & Apocynaceae & Tinaikibo & 1.05 & 61.7 & Forest & $\mathrm{Ar}$ & - \\
\hline Agave sisalana Perrine & Agavaceae & Lalohasy & 1.04 & 19.6 & Forest & $L x$ & - \\
\hline Commiphora simplicifolia H. Perrier & Burseraceae & Sengatse & 1.04 & 10.6 & Forest & $\operatorname{Ar}$ & Z.S. Rogers 870 \\
\hline Hippocratea angustipetala H. Perrier & Celastraceae & Vahimpindy & 1.04 & 11.1 & Forest & $\operatorname{Ar}$ & - \\
\hline Musa sp. & Musaceae & Kida & 1.04 & 46.8 & Crop field & $\mathrm{Fr}$ & - \\
\hline Pentopetia androsaemifolia Decne. & Apocynaceae & Ntsompia & 1.04 & 9.8 & Crop field, Fallow & LV & Arne Anderberg 123 \\
\hline Strychnos sp. 2 & Loganiaceae & Mangerivorika & 1.04 & 19.6 & Forest & $\mathrm{Ar}$ & - \\
\hline Tridax procumbens $\mathrm{L}$. & Asteraceae & Angamay & 1.04 & 53.6 & Crop field, Fallow & LV & P.B. Phillipson 1791 \\
\hline Uncarina stellulifera Humbert & Pedaliaceae & Farehitse & 1.04 & 9.8 & Forest & LV & P.B. Phillipson 2723 \\
\hline
\end{tabular}


Table 3 List of medicinal plants species used in the Mahafaly region, SW-Madagascar (Continued)

\begin{tabular}{|c|c|c|c|c|c|c|c|}
\hline Delonix floribunda (Baill.) Capuron & Fabaceae & Fengoky & 1.03 & 40 & Forest & $L x$ & J. Bosser 13584 \\
\hline Jatropha curcas L. & Euphorbiaceae & Savoa & 1.03 & 39.2 & Forest & $\mathrm{Lv}, \mathrm{Sb}, \mathrm{Lx}$ & P.B. Phillipson 1725 \\
\hline Loeseneriella rubiginosa (H. Perrier) N. Hallé & Celastraceae & Timbatse & 1.03 & 35.7 & Forest & LV & B. Du puy MB 570 \\
\hline Terminalia ulexoides H. Perrier & Combretaceae & Fatra & 1.03 & 13.6 & Forest & $\mathrm{Sb}$ & L. J. Dorr 4057 \\
\hline Androya decaryi H.Perrier & Scrophulariaceae & Manateza & 1.02 & 23 & Forest & LV & $\begin{array}{l}\text { Herbier du Laboratoire de } \\
\text { Botanique } 1777\end{array}$ \\
\hline Fernandoa madagascariensis (Baker) A.H. Gentry & Bignoniaceae & Somontsoy & 1.02 & 46.8 & Forest & $\mathrm{Lv}, \mathrm{Br}$ & L.J. Dorr 3960 \\
\hline Ocimumcanum Sims. & Lamiaceae & Romberombe & 1.02 & 37.9 & Forest & $\operatorname{Ar}$ & B. Croat 31282 \\
\hline Tabernaemontana sp. & Apocynaceae & Feka & 1.01 & 40.4 & Forest & $\mathrm{Sb}$ & - \\
\hline Zanthoxylum tsihanimposa H.Perrier & Rutaceae & Manongo & 1.01 & 60 & Forest & $\mathrm{Sb}$ & P. Morat 4677 \\
\hline Abrus precatorius $\mathrm{L}$. & Fabaceae & Voamena & 1 & 2.6 & Forest & $\operatorname{Ar}$ & J. Bosser 19395 \\
\hline Acacia farnesiana (L.) Willd. & Fabaceae & Kasy & 1 & 1.7 & Savanna & $\operatorname{Ar}$ & D.J. \& B.P. Dupuy M69 \\
\hline Acacia sp. 5 & Fabaceae & Anadrohy & 1 & 0.4 & Forest & $\mathrm{Br}$ & - \\
\hline Acacia viguieri Villiers \& Du Puy & Fabaceae & Roybenono & 1 & 3 & Forest & Ar & H. Humbert 2487 \\
\hline Adansonia rubrostipa Jum. \& H.Perrier & Malvaceae & Fony & 1 & 2.6 & Forest & $\mathrm{Fr}$ & J. Bosser 15743 \\
\hline Adansonia za Baill. & Malvaceae & Zan & 1 & 4.3 & Forest & $\mathrm{Fr}$ & P.B. Phillipson 2638 \\
\hline Aerva javanica (Burm. f.) Juss. & Amaranthaceae & Volofoty & 1 & 6 & Forest & $\mathrm{Sb}$ & M.R. Decary 18863 \\
\hline Alantsilodendron alluaudianum (R.Vig.) Villiers & Fabaceae & Havoa & 1 & 0.4 & Forest & $\operatorname{Ar}$ & - \\
\hline Albizia bernieri E. Fourn. ex Villiers & Fabaceae & Halimboro & 1 & 2.1 & Forest & $\mathrm{Br}$ & P.B. Phillipson 5285 \\
\hline Albizia tulearensis R.Vig. & Fabaceae & Mendoravy & 1 & 0.4 & Forest & $\mathrm{Br}$ & D.J. \& B. P. Dupuy M54 \\
\hline Allium sativum $\mathrm{L}$. & Amaryllidaceae & Tongologasy & 1 & 5.5 & Crop field & $\mathrm{Sb}$ & - \\
\hline Aloe antandroi (R.Decary) H. Perrier & Xanthorrhoeaceae & Sotry & 1 & 2.1 & Forest & LV & M.R. Decary 9886 \\
\hline Alysicarpus vaginalis (L.) D.C. & Fabaceae & Tokampototse & 1 & 6.4 & Crop field, Fallow & Ar & Thomas B. Croat 31195 \\
\hline Amaranthus viridis $\mathrm{L}$. & Amaranthaceae & Beamena & 1 & 0.4 & Crop field, Fallow & Ar & - \\
\hline Anisotes madagascariensis Benoist & Acanthaceae & Hazontsoy & 1 & 1.3 & Forest & $\operatorname{Ar}$ & Rauh 1097 \\
\hline Arachis hypogaea $\mathrm{L}$. & Fabaceae & Kapiky & 1 & 17.5 & Crop field & $\mathrm{Fr}$ & - \\
\hline Asparagus calcicola H. Perrier & Asparagaceae & Fio & 1 & 0.4 & Forest, Fallow & $\mathrm{Sb}$ & J. Bosser 10599 \\
\hline Azadirachta indica A. Juss. & Meliaceae & Nimo & 1 & 6.4 & Forest & LV & Armand Rakotozafy 1798 \\
\hline Barleria brevituba Benoist & Acanthaceae & Patipatikantala & 1 & 0.4 & Savanna, Fallow & Ar & P. Morat 627 \\
\hline Bathiorhamnus cryptophorus Capuron & Rhamnaceae & Losy & 1 & 11.5 & Forest & $\mathrm{Sb}$ & - \\
\hline Berchemia discolor (Klotzsch) Hemsl. & Rhamnaceae & Vorodoke & 1 & 1.7 & Forest & Ar & - \\
\hline Calopyxis grandidieri (Drake) Capuron ex Stace & Combretaceae & Tsambara & 1 & 1.7 & Forest & $\mathrm{Fr}$ & B Lewis 1294 \\
\hline Capsicum sp. & Solanaceae & Sakay & 1 & 21.3 & Crop field & $\mathrm{Fr}$ & \\
\hline Capurodendron androyense Aubrév. & Sapotaceae & Nato & 1 & 11.5 & Forest & $\mathrm{Sb}, \mathrm{Br}$ & J. Bosser 10352 \\
\hline
\end{tabular}


Table 3 List of medicinal plants species used in the Mahafaly region, SW-Madagascar (Continued)

\begin{tabular}{|c|c|c|c|c|c|c|c|}
\hline Carica papaya L. & Caricaceae & Papaye & 1 & 6 & Crop field & LV & Herbier du Jardin Botanique 324 \\
\hline Carissa spinarum $\mathrm{L}$. & Apocynaceae & Lamontindahy & 1 & 0.4 & Forest & $\operatorname{Ar}$ & - \\
\hline Chadsia grevei Drake & Fabaceae & Sanganakoholahy & 1 & 7.7 & Forest & $\operatorname{Ar}$ & D.J. \& B.P. Dupuy M38 \\
\hline Chamaesyce hirta (L.) Millsp. & Euphorbiaceae & Kimenamena & 1 & 7.7 & Crop field & Lv & Robert W. Brooks 8 \\
\hline Citrus medica L. & Rutaceae & Tsoha & 1 & 0.4 & Crop field & $\mathrm{Sb}$ & - \\
\hline Cocos nucifera $\mathrm{L}$. & Arecaceae & Voanio & 1 & 0.4 & Seaside & $\mathrm{Fr}$ & - \\
\hline Colvillea racemosa Bojer & Fabaceae & Sarongaza & 1 & 14 & Forest & $\mathrm{Br}$ & P.B. Phillipson 2802 \\
\hline Commiphora humbertii H. Perrier & Burseraceae & Andrambely & 1 & 0.4 & Forest & Lv & S. Eboroke 870 \\
\hline Commiphora marchandii Engl. & Burseraceae & Vingovingo & 1 & 0.4 & Forest & $\operatorname{Ar}$ & James S. Miller 6160 \\
\hline Cordia caffra Sond. & Boraginaceae & Varo & 1 & 1.7 & Forest & Lv & Thomas B .Croat 30787 \\
\hline Crinum asiaticum L. & Amaryllidaceae & Tongolondolo & 1 & 0.4 & Forest & $\mathrm{Sb}$ & - \\
\hline Crotalaria androyensis R. Vig. & Fabaceae & Katsankantsa & 1 & 0.9 & Forest & $\operatorname{Ar}$ & M.R. Decary 9517 \\
\hline Crotalaria fiherenensis R.Vig. & Fabaceae & Voniloha & 1 & 0.9 & Savanna, Forest, Fallow & $\operatorname{Ar}$ & - \\
\hline Croton catatii Baill. & Euphorbiaceae & Somorombohitse & 1 & 0.9 & Forest & $\operatorname{Ar}$ & M.R. Decary 10495 \\
\hline Cryptostegia madagascariensis Bojer ex Decne & Apocynaceae & Lombiry & 1 & 4.7 & Forest & $\mathrm{Lv}, \mathrm{Sb}$ & P.B. Phillipson 2622 \\
\hline Cucurbita maxima Duch. & Cucurbitaceae & Trehaky & 1 & 0.4 & Crop field & $\operatorname{Ar}$ & J.Bosser 13577 \\
\hline Cymbopogon citratus (DC.) Stapf & Poaceae & Veromanitse & 1 & 0.4 & Crop field & $\operatorname{Ar}$ & - \\
\hline Cynodon dactylon (L.) Pers. & Poaceae & Kidresy & 1 & 4.7 & Forest & $\operatorname{Ar}$ & J. Bosser 10540 \\
\hline Cyphostemma amplexicaule Desc. & Vitaceae & Tahezantrandrake & 1 & 1.3 & Forest & Lv & J. Bosser 19194 \\
\hline Dicoma incana (Baker) O. Hoffm. & Asteraceae & Peha & 1 & 10.2 & Forest & $\mathrm{Sb}$ & P.B. Phillipson 2426 \\
\hline Dicraeopetalum mahafaliense (M.Pelt.) Yakovlev & Fabaceae & Lovainafy & 1 & 1.7 & Forest & $\mathrm{Br}$ & Thomas B. Croat 30969 \\
\hline Dioscorea bemandry Jum. \& H. Perrier & Dioscoreaceae & Baboke & 1 & 0.4 & Forest & $\mathrm{Sb}$ & L.R. Caddick 339 \\
\hline Dioscorea fandra H. Perrier & Dioscoreaceae & Andraha & 1 & 2.1 & Forest & $\mathrm{Sb}$ & Gordon McPherson 17451 \\
\hline Dioscorea nako H. Perrier & Dioscoreaceae & Fandra & 1 & 0.4 & Forest & $\mathrm{Sb}$ & L.R. Caddick 331 \\
\hline Dioscorea ovinala Baker & Dioscoreaceae & Behandaliny & 1 & 0.9 & Forest & $\operatorname{Ar}$ & J.N. Labat 2111 \\
\hline $\begin{array}{l}\text { Diospyros tropophylla (H. Perrier) G.E. Schatz } \\
\text { \& Lowry }\end{array}$ & Ebenaceae & Remeloky & 1 & 2.1 & Forest & $\operatorname{Ar}$ & P. Morat 565 \\
\hline Ehretia decaryi J. S. Mill. & Boraginaceae & Lampana & 1 & 6 & Forest & $\operatorname{Ar}$ & J. Bosser 10116 \\
\hline Enterospermum madagascariense (Baill.) Homolle & Rubiaceae & Masonjoany & 1 & 0.4 & Forest & $\operatorname{Tr}$ & - \\
\hline Erythrophysa aesculina Baill. & Sapindaceae & Handimbohitse & 1 & 2.6 & Forest & $\operatorname{Ar}$ & G.E. Schatz 1777 \\
\hline Euclinia suavissima (Homolle ex Cavaco) J.-F. Leroy & Rubiaceae & Voafotaky & 1 & 0.9 & Forest & $\mathrm{Fr}$ & J. Bosser 13353 \\
\hline Euphorbia arahaka Poisson & Euphorbiaceae & Samatafoty & 1 & 14.9 & Savanna, Forest, crop field & LV & M.D. Decary 3008 \\
\hline Ficus polita Vahl & Moraceae & Aviavy & 1 & 3.8 & Forest & $\mathrm{Br}$ & M.R. Decary 5031 \\
\hline Ficus sp. & Moraceae & Nonoka & 1 & 1.7 & Fallow, Forest & $\mathrm{Br}$ & - \\
\hline
\end{tabular}

Cyphostemma amplexicaule Desc.

Dioscorea bemandry Jum. \& H. Perrier

Dioscorea ovinala Baker

Diospyros tropophylla (H. Perrier) G. S Schatz

Ehretia decaryi J. S. Mill.

Sapindaceae

Euphorbiaceae

Fallow, Forest 
Table 3 List of medicinal plants species used in the Mahafaly region, SW-Madagascar (Continued)

\begin{tabular}{|c|c|c|c|c|c|c|c|}
\hline Ficus trichopoda Baker & Moraceae & Fihamy & 1 & 39.2 & Forest & $\operatorname{Tr}$ & S.T. Malcomber 1116 \\
\hline Flacourtia indica (Burm. f.) Merr. & Salicaceae & Lamonty & 1 & 3.8 & Forest & $\mathrm{Sb}, \mathrm{Fr}$ & C.C.H. Jongkind 3720 \\
\hline Gnidia linearis (Leandri) Z.S. Rogers & Thymeleaceae & Ronisa & 1 & 1.3 & Forest & Lv & Z.S. Rogers 930 \\
\hline Gonocrypta grevei (Baill.) Costantin \& Gallaud & Apocynaceae & Piravola & 1 & 6.8 & Forest & $L x$ & P.B. Phillipson 1669 \\
\hline Gossypium arboreum L. & Malvaceae & Hasy & 1 & 3.8 & Crop field, Fallow & Lv & H. Humbert 5166 \\
\hline Grewia grevei Baillon & Malvaceae & Tombokampaha & 1 & 0.9 & Forest & $\operatorname{Ar}$ & J. Bosser 19338 \\
\hline Grewia microcyclea (Burret) Capuron \& Mabb. & Malvaceae & Hazofoty & 1 & 3.8 & Forest & $\mathrm{Br}$ & Jacqueline \& M. Peltier 1285 \\
\hline Helinus integrifolius (Lam.) Kuntze & Rhamnaceae & Masokarany & 1 & 2.1 & Forest & $\operatorname{Ar}$ & P.B. Phillipson 1737 \\
\hline Indigofera tinctoria $\mathrm{L}$. & Fabaceae & Sarikapiky & 1 & 49.4 & Fallow, Savanna & $\mathrm{Ar}$ & J.N. Labat 2104 \\
\hline Ipomea sp. 1 & Convolvulaceae & Sarivelahy & 1 & 1.7 & Forest, Savanna, Fallow & Lv & - \\
\hline Ipomea sp. 2 & Convolvulaceae & Velahy & 1 & 1.3 & Forest & $L x$ & - \\
\hline Kalanchoe beharensis Drake & Crassulaceae & Mongy & 1 & 0.4 & Forest & Lv & James L. Zarucchi 7471 \\
\hline Kalanchoe sp. & Crassulaceae & Relefo & 1 & 3.4 & Forest & Lv & - \\
\hline Karomia microphylla (Moldenke) R.B. Fern. & Lamiaceae & Forimbitika & 1 & 0.9 & Forest & $\mathrm{Br}$ & P.B. Phillipson 3438 \\
\hline Kleinia madagascariensis (Humbert) P. Hallyday & Asteraceae & Malaohira & 1 & 2.6 & Forest & $\mathrm{Ar}$ & P.B. Phillipson 2475 \\
\hline $\begin{array}{l}\text { Koehneria madagascariensis (Baker) S.A. Graham, } \\
\text { Tobe \& Baas }\end{array}$ & Lythraceae & Fizolotsora & 1 & 1.7 & Forest & $\operatorname{Ar}$ & L.J. Dorr 4063 \\
\hline Lablab purpureus (L.) Sweet & Fabaceae & Antaky & 1 & 9.4 & Crop field & $\mathrm{Fr}$ & Michelle Sauther 27 \\
\hline Leucosalpa grandiflora Humbert & Orobanchaceae & Tamborisahy & 1 & 1.7 & Forest & $\mathrm{Sb}$ & P. Morat 2978 \\
\hline Maerua filiformis Drake & Capparaceae & Somangy & 1 & 1.3 & Forest & Lv,Ar & P.B. Phillipson 2417 \\
\hline Maerua nuda Scott-Elliot & Capparaceae & Somangilahy & 1 & 1.7 & Forest & Lv & J. Bosser 10507 \\
\hline Manihot esculenta Crantz & Euphorbiaceae & Balahazo & 1 & 8.1 & Crop field & $\mathrm{Lv}, \mathrm{Sb}$ & - \\
\hline Margaritaria anomala (Baill.) Fosberg & Phyllanthaceae & Tsivano & 1 & 18.7 & Forest & $\mathrm{Sb}$ & - \\
\hline Marsdenia cordifolia Choux & Apocynaceae & Bokabe & 1 & 2.6 & Forest & $L x$ & P.B. Phillipson 2741 \\
\hline Mundulea stenophylla R. Vig. & Fabaceae & Rodrotse & 1 & 1.7 & Forest & Lv & M.R. Decary 2527 \\
\hline Olax andronensis Baker & Olacaceae & Bareraky & 1 & 0.4 & Forest & $\mathrm{Sb}$ & L.J. Razafintsalama 785 \\
\hline Opuntia monacantha Haw. & Cactaceae & Notsoky & 1 & 2.6 & Fallow, Savanna & $\mathrm{Fr}$ & - \\
\hline Pachypodium geayi Costantin \& Bois & Apocynaceae & Vontake & 1 & 0.4 & Forest & $\operatorname{Tr}$ & P.B Phillipson 2610 \\
\hline Panicum pseudowoeltzkowii A. Camus & Poaceae & Ahikitoto & 1 & 0.4 & Forest & Lv & J. Bosser 308 \\
\hline Panicum sp. & Poaceae & Mandavohita & 1 & 0.4 & Fallow, Forest, Savanna & $\operatorname{Ar}$ & - \\
\hline Persea americana Mill. & Lauraceae & Zavoka & 1 & 0.9 & Crop field & $\mathrm{Fr}$ & - \\
\hline Pervillaea phillipsonii Klack. & Apocynaceae & Sangisangy & 1 & 0.4 & Forest & $\operatorname{Ar}$ & P.B. Phillipson 3472 \\
\hline Phaseolus lunatus L. & Fabaceae & Kabaro & 1 & 5.5 & Crop field & $\mathrm{Fr}$ & J. Bosser 1011 \\
\hline Phyllanthus casticum Willemet & Phyllanthaceae & Sanira & 1 & 6 & Forest & Lv & P.B. Phillipson 2392 \\
\hline
\end{tabular}


Table 3 List of medicinal plants species used in the Mahafaly region, SW-Madagascar (Continued)

\begin{tabular}{|c|c|c|c|c|c|c|c|}
\hline Plumbago aphylla Bojer ex Boiss. & Plumbaginaceae & Motemote & 1 & 1.7 & Forest & $\operatorname{Ar}$ & H. Humbert 19960 \\
\hline Poupartia minor (Bojer) L. Marchand & Anacardiaceae & Sakoakomoky & 1 & 2.1 & Forest & $\mathrm{Br}$ & P.B. Phillipson 1813 \\
\hline Psidium sp. & Myrtaceae & Goavy & 1 & 0.4 & Crop field, Fallow & LV & - \\
\hline Radamaea montana Benth. & Orobanchaceae & Tamotamo & 1 & 31.5 & Forest & $\mathrm{Sb}$ & J. Bosser 6071 \\
\hline Rhopalopilia hallei Villiers & Opiliaceae & Malainevotsy & 1 & 11.5 & Forest & $\mathrm{Ar}$ & - \\
\hline Ricinus communis L. & Euphorbiaceae & Kinana & 1 & 5.5 & Crop field, Fallow & LV & Thomas B. Croat 28615 \\
\hline Roupellina boivinii (Baill.) Pichon & Apocynaceae & Lalondo & 1 & 0.9 & Forest & Lv & - \\
\hline Secamone geayi Costantin \& Gallaud & Apocynaceae & Kililo & 1 & 4.7 & Forest & $\operatorname{Ar}$ & J. Bosser 15917 \\
\hline Strychnos madagascariensis Poir. & Loganiaceae & Bakoa & 1 & 7.7 & Forest & $\mathrm{Sb}, \mathrm{Fr}$ & J. Bosser 14492 \\
\hline Tephrosia purpurea (L.) Pers. & Fabaceae & Engetsengetse & 1 & 5.1 & Forest & Lv & Jacqueline \& M. Peltier 9936 \\
\hline Terminalia disjuncta H. Perrier & Combretaceae & Taly & 1 & 1.7 & Forest & $\operatorname{Ar}$ & B. Dupuy 629 \\
\hline Trema orientalis (L.) Blume & Cannabaceae & Andrarezona & 1 & 0.4 & Forest & $\operatorname{Tr}$ & B. Lewis 1292 \\
\hline Typha angustifolia L. & Typhaceae & Vondro & 1 & 0.4 & Forest & LV & M.R. Decary 14868 \\
\hline Vigna unguiculata (L.) Walp. & Fabaceae & Loji & 1 & 20.4 & Crop field & $\mathrm{Fr}$ & Thomas B. Croat 32050 \\
\hline $\begin{array}{l}\text { Xerophyta tulearensis (H. Perrier) Phillipson } \\
\text { \& Lowry }\end{array}$ & Velloziaceae & Tsimatefaosa & 1 & 0.4 & Forest & $\mathrm{Ar}$ & P.B Phillipson 2459 \\
\hline Xerosicyos danguyi Humbert & Cucurbitaceae & Tapisaky & 1 & 1.3 & Forest & LV & Thomas B. Croat 30795 \\
\hline Ziziphus mauritiana Lam. & Rhamnaceae & Konazy & 1 & 0.4 & Savanna & $\mathrm{Br}$ & D. Seigler 12891 \\
\hline Ziziphus mucronata Willd. & Rhamnaceae & Tsinefonala & 1 & 4.7 & Forest & $\mathrm{Br}$ & Harb. Inst. Sci. Mad. 4517 \\
\hline
\end{tabular}

$\mathrm{L} v=$ Leaves, $\mathrm{Ar}=$ Aerial parts, $\mathrm{Sb}=$ Subterranean parts, $\mathrm{Fr}=$ Fruits or seeds, $\mathrm{Lx}=\mathrm{Sap}$ or latex, $\mathrm{Tr}=\mathrm{Trunk}, \mathrm{St}=\mathrm{Stems}, \mathrm{Br}=$ stem barks; $\left.{ }^{*}\right)$ Voucher number represents the number of the specimens from which our plants were determined in Tsimbazaza Herbarium, Madagascar. 


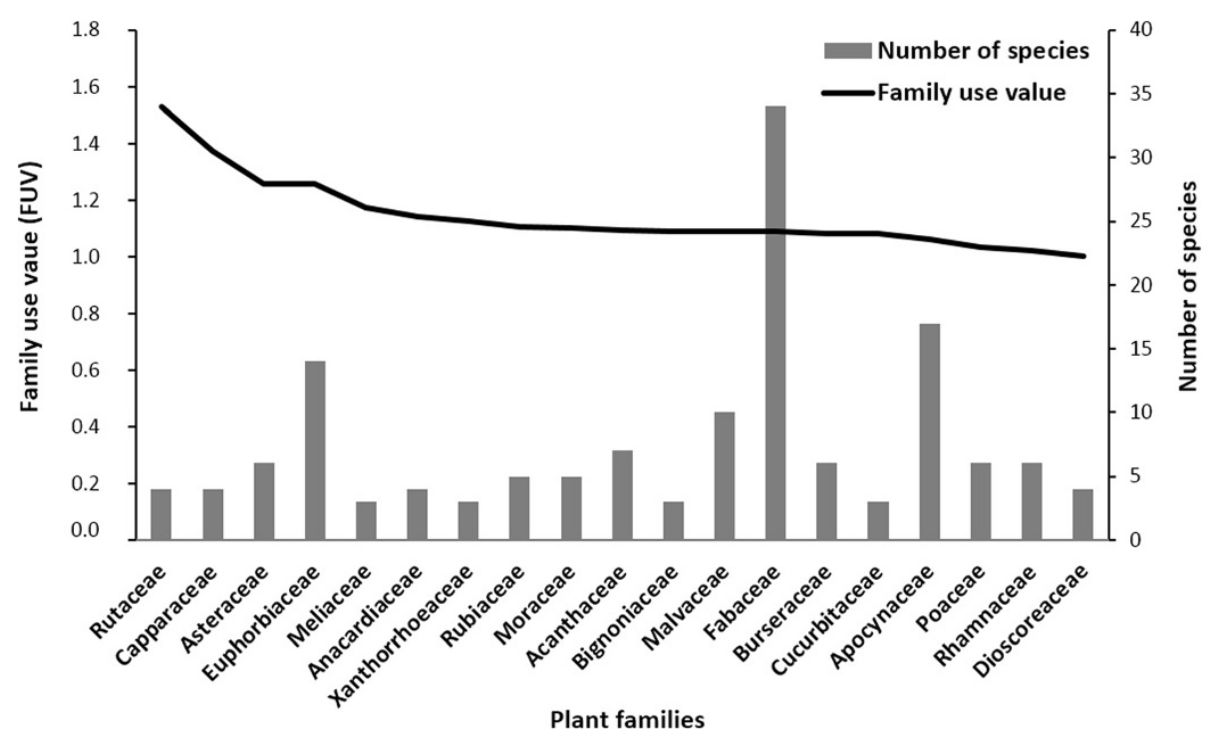

Figure 2 Most important plant families identified by family use value (FUV, description see Table 1) and number of medicinal plant species per family used in the Mahafaly region in SW-Madagascar.

disorders, muscular skeletal problems and cosmetic care for women.

The growth forms of the recorded plants species are shrubs $(38 \%)$, trees $(28 \%)$, herbs $(20 \%)$, lianas $(11 \%)$, vines $(2 \%)$, and epiphytes (less than 1\%; Figure 3A). Most medicinal plants $(82 \%)$ are collected in forest areas, $14 \%$ are cultivated and the rest is typically found in fallow land or rangelands such as bushland and grassland. Although the majority of the used plants are endemic to Madagascar (68\%), exotic plants or plants that have a large worldwide distribution are used as well. Altogether, $95 \%$ of the recorded medicinal plants can be found in the Mahafaly region, the remainder are species bought or imported from the nearest town or from neighbouring regions.

The most frequently collected plant parts are the aboveground plant material (i.e., stems and leaves, 25\%), leaves $(23 \%)$ and subterranean parts (roots and tubers, $20 \%$; Figure $3 \mathrm{~B}$ ). Single stems are not often used for medicinal purposes $(2 \%)$, whereas the roots of plants are used, especially for post-delivery treatment, women genital and cosmetic care, such as Ximenia perrieri ('Kotro'). Sometimes people use different parts of the same plant, especially if it has a high use value (i.e. used for different medicinal purposes), such as Neobeguea mahafaliensis ('Handy'). The stem barks of this species are used to treat muscular-skeletal problems and its below ground parts serve women during the post-delivery process.

Regarding the use of species, Aloe divaricata (used by $100 \%$ of informants), Cedrelopsis grevei $(100 \%)$ and Neobeguea mahafaliensis (91\%) predominate. Aloe divaricata is a locally important species with 28 different uses. Altogether, 46 types of medicinal uses were reported (Cook [31]; Table 4). Some species, such as Operculicarya decaryi, may also be used in multiple ways such as a body tonic, for women genital care and to alleviate nutritional

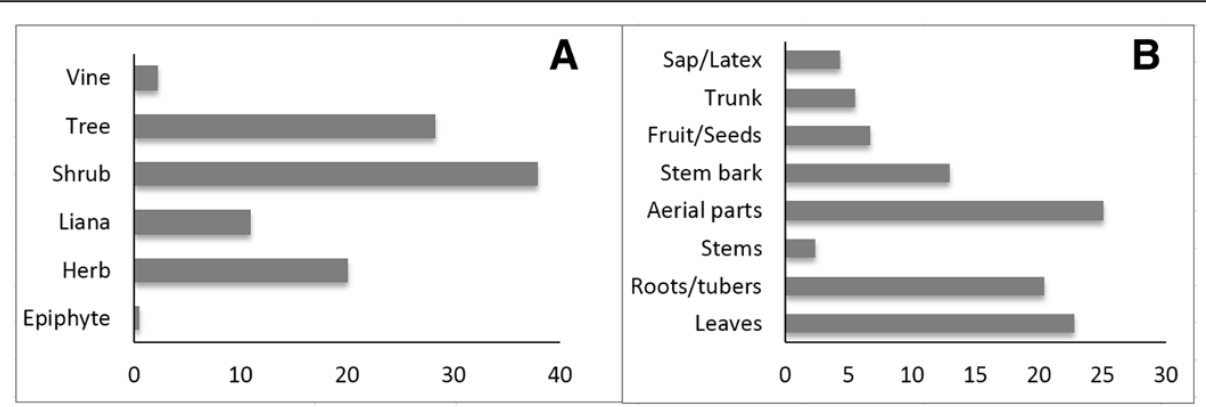

Figure 3 Proportion of life forms used as medicinal plants (A); Proportion of plant parts used for traditional healing (B) in the Mahafaly region of SW-Madagascar. 
Table 4 Categories of diseases and their respective most cited plant species in the Mahafaly region of SW Madagascar

\begin{tabular}{|c|c|}
\hline Diseases and use category & Most cited species \\
\hline Digestive disorders & Aloe divaricata A. Berger, Cedrelopsis grevei Baill. \\
\hline Muscular_Skeletal & Neobeguea mahafaliensis J.-F. Leroy, Cedrelopsis grevei Baill. \\
\hline Eye problems & Tamarindus indica L., Jatropha mahafalensis Jum. \& H. Perrier, Fernandoa madagascariensis (Baker) A.H. Gentry \\
\hline Wound/Injury/Swelling & Tridax procumbens L., Tabernaemontana sp., Croton geayi Leandri \\
\hline Ear infections & Citrullus lanatus (thunb.) Matsum. \& Nakai, Cynanchum grandidieri Liede \& Meve \\
\hline Flue/Fever & Ocimum canum Sims., Croton geayi Leandri \\
\hline Skin disorders & Lemuropisum edule H. Perrier \\
\hline Post delivery care & Erythroxylum retusum Baill. ex O.E. Schulz, Salvadora angustifolia Turill, Loeseneriella rubiginosa (H. Perrier) N. Hallé \\
\hline Toothache & Zanthoxylum tsihanimposa H.Perrier, Euphorbia tirucalli L. \\
\hline Venereal infections & Cynodon dactylon (L.) Pers., Euphorbia tirucalli L., Blepharis calcitrapa Benoist \\
\hline Respiratory system disorders & Cynanchum perrieri Choux, Indigofera compressa Lam. \\
\hline Malaria & Cajanus cajan (L.) Millsp., Indigofera tinctoria L. \\
\hline Sprains & Aloe divaricata A.Berger, Delonix floribunda (Baill.) Capuron \\
\hline New born care & Coffea grevei Drake ex A. Chev, Pentatropis nivalis subsp. madagascariensis (Decne.) Liede \& Meve \\
\hline Circulatory system disorders & Opuntia sp. (Raketamena) \\
\hline Woman genital hygiene & Ximenia perrieri Cavaco \& Keraudren, Operculicarya decaryi H. Perrier, Cedrelopsis grevei Baillon \\
\hline Cosmetic/Hair care & Ficus trichopoda Baker, Cedrelopsis grevei Baill. \\
\hline Body tonic & Erythroxylum retusum Baill. ex O.E. Schulz, Neobeguea mahafaliensis J.-F. Leroy, Operculicarya decaryi H. Perrier \\
\hline Nutritional disorders & Tamarindus indica L., Adansonia za Baill., Operculicarya decaryi H. Perrier \\
\hline Livestock disease & Vigna unguiculata (L.) Walp. \\
\hline
\end{tabular}

disorders during famine periods. Tamarindus indica was used to treat eye problems, but it is similarly important to alleviate nutritional disorders.

Apparently digestive system disorders (13\%), wound and injury problems (12\%) and post-delivery care for women (11\%) represented the most prevalent health problems in the study area. The use of medicinal plants in cosmetic and genital care of women amounted to $8 \%$, similar to plant use for 'body tonic' after hard physical work.

\section{Plant uses and knowledge patterns among households}

Based on their socio-economic characteristics and the use intensity of forest products, the cluster analysis revealed two groups of households (Table 5). The well-off farmers represent households with a high number of livestock, off-farm activities and a higher education level. They use yam as a supplementary food, practice a more sustainable harvest technique and collect less wild yam tubers compared with the poorer farmers. The latter are characterized by lower household assets and off-farm activities. Farmers of this group collect more yam species and use their tubers as staple food.

Most of the socio-economic variables used for the cluster analysis were effective in discriminating the two defined household groups except for the education level and the diversity of medicinal plant use. Together the predictors accounted for $51 \%$ of the between-group variability. Based on the conclusions of Rach et al. that structure coefficients $\geq 0.30$ indicate a strong discriminating power [39], households cluster groups were determined by the amount of agricultural harvest, livestock owned by household, and the frequency of wild yams collection. In contrast, the number of medicinal plants used and the use intensity of medicinal plants differed only slightly among the two groups.

\section{Plant uses and knowledge patterns among villages}

Collection and use of forest plants differed between the littoral (Efoetse) and the plateau (the other three villages) which may be mainly explained by the lack of forest resources and wild yams in the coastal area. The number of medicinal plants and wild yam species used were higher on the plateau (Ampotake, Andremba, Itomboina, Miarintsoa), and the number of species collected was highest in Itomboina and Miarintsoa (Table 6). However, the collection frequency, period, and the amount of harvested wild yam were higher in Ampotake. This may be mainly due to the proximity of community based forests, where collection of forest products is not restricted. Itomboina and Miarintsoa are situated in the middle of the plateau, where different soil types (ferralitic, red sandy and calcareous soils) and forest habitats prevail, which may explain the high diversity in species collection 
Table $\mathbf{5}$ Results of two step cluster and discriminant analysis of $\mathbf{2 5 0}$ interviewed rural households in the Mahafaly Region of SW-Madagascar

\begin{tabular}{|c|c|c|c|c|c|}
\hline \multirow[t]{3}{*}{ Selected variables } & \multicolumn{2}{|c|}{ Cluster group } & \multicolumn{3}{|c|}{ Discriminant analysis } \\
\hline & \multirow{2}{*}{$\begin{array}{l}\text { Well-off farmers } \\
\text { Mean } \pm \text { SD }\end{array}$} & \multirow{2}{*}{$\begin{array}{c}\text { Less well-off farmers } \\
\text { Mean } \pm \text { SD* }^{*}\end{array}$} & & & \\
\hline & & & Wilks' Lambda & Sig & Structure coefficients \\
\hline Education level & $1.03 \pm 0.71$ & $0.86 \pm 0.69$ & 0.986 & 0.068 & 0.116 \\
\hline Agricultural harvest & $1.23 \pm 0.42$ & $0.52 \pm 0.63$ & 0.747 & $0.000^{* *}$ & 0.574 \\
\hline Households activities & $1.11 \pm 0.71$ & $0.26 \pm 0.44$ & 0.928 & $0.000^{* *}$ & 0.274 \\
\hline Family size & $7.35 \pm 3.55$ & $6.4 \pm 3.20$ & 0.982 & $0.037^{*}$ & 0.133 \\
\hline Tropical livestock unit ${ }^{1)}$ & $12.53 \pm 12.32$ & $2.18 \pm 5.40$ & 0.746 & $0.000^{* *}$ & 0.577 \\
\hline Agricultural area & $2.86 \pm 2.30$ & $1.19 \pm 1.60$ & 0.945 & $0.000^{* *}$ & 0.239 \\
\hline Medicinal plants used & $27.77 \pm 13.55$ & $32.7 \pm 14.30$ & 0.974 & $0.011^{*}$ & -0.162 \\
\hline Number of medicinal uses & $13.87 \pm 4.27$ & $15.6 \pm 3.60$ & 0.976 & $0.016^{*}$ & -0.153 \\
\hline Diversity of medicinal plant use & $23.35 \pm 2.12$ & $25.92 \pm 2.10$ & 0.988 & 0.089 & -0.108 \\
\hline Wild yam species collected & $2.23 \pm 2.71$ & $3.17 \pm 2.17$ & 0.960 & $0.002^{* *}$ & -0.201 \\
\hline Yam tubers harvested per collection event ${ }^{2)}$ & $6.72 \pm 6.74$ & $13.02 \pm 10.33$ & 0.908 & $0.000^{* *}$ & -0.314 \\
\hline Frequency of collection & $2.35 \pm 2.71$ & $5.83 \pm 5.23$ & 0.886 & $0.000^{* *}$ & -0.354 \\
\hline Sale & $3.95 \pm 11.09$ & $17.03 \pm 24.12$ & 0.920 & $0.000^{* *}$ & -0.291 \\
\hline Collection period & $2.40 \pm 2.29$ & $13.78 \pm 2.79$ & 0.943 & $0.000^{* *}$ & -0.243 \\
\hline \multirow[t]{3}{*}{ Use of wild yams } & $1.73 \pm 0.44$ & $1.49 \pm 0.50$ & 0.948 & $0.000^{* *}$ & 0.231 \\
\hline & & & \multicolumn{3}{|c|}{ Eigen Value $=1.026$} \\
\hline & & & \multicolumn{3}{|c|}{ Percentage variance $=50.41$} \\
\hline
\end{tabular}

${ }^{1)}[38]{ }^{2)}$ Number of harvest holes per collection event, ${ }^{*}$ significance level at $p \leq 0.05,{ }^{*}$ significance level at $p \leq 0.01$.

Table 6 Descriptive statistics of variables (Mean \pm SD) used in evaluating the knowledge and uses of wild yams and medicinal plants of the Mahafaly region in SW-Madagascar

\begin{tabular}{|c|c|c|c|c|c|}
\hline Variables & Ampotake $(n=50)$ & Andremba $(n=50)$ & Itomboina $(n=50)$ & Miarintsoa $(n=50)$ & Efoetse $(n=50)$ \\
\hline \multicolumn{6}{|l|}{ Collection of wild yams (\%): } \\
\hline D. alatipes & 92.16 & 80.3 & 80 & 42 & 0 \\
\hline D. bemandry & 94.12 & 51.52 & 80 & 87.23 & 0 \\
\hline D. fandra & 54.9 & 60.61 & 60 & 59.57 & 0 \\
\hline D. ovinala & 76.47 & 62.12 & 64.44 & 46.81 & 0 \\
\hline D. nako & 43.14 & 21.21 & 66.67 & 48.94 & 0 \\
\hline D. soso & 7.84 & 39.39 & 46.67 & 21.28 & 0 \\
\hline Number of wild yams species collected & $3.9 \pm 1.1$ & $3.9 \pm 1.3$ & $4.2 \pm 1.4$ & $4.9 \pm 1.9$ & 0 \\
\hline Frequency of wild yams collection ${ }^{1)}$ & $9.8 \pm 5.7$ & $5.1 \pm 2.5$ & $5.6 \pm 2.9$ & $5.7 \pm 3.9$ & 0 \\
\hline Period of collection (months/year) & $5.7 \pm 1.9$ & $4.1 \pm 1.9$ & $4.2 \pm 1.4$ & $4.9 \pm 1.9$ & 0 \\
\hline Wild yams harvested ${ }^{2)}$ & $21 \pm 9$ & $12.8 \pm 5.8$ & $14.1 \pm 5.6$ & $13.1 \pm 7.6$ & 0 \\
\hline Unsustainable harvest technique (\%) & 89.6 & 81.5 & 89.5 & 78.6 & - \\
\hline Sustainable harvest technique (\%) & 10.4 & 18.5 & 10.5 & 21.4 & - \\
\hline Monthly income, from wild yams (US\$) & $5.5 \pm 7.4$ & $1.3 \pm 3.5$ & $2.0 \pm 3.0$ & $1.3 \pm 2.5$ & 0 \\
\hline Number of medicinal species used & $43.5 \pm 12$ & $29.8 \pm 11.8$ & $36.6 \pm 10$ & $27.4 \pm 12.4$ & $18.4 \pm 9.7$ \\
\hline Diversity of medicinal plant use & $33.5 \pm 10.3$ & $23.9 \pm 8.6$ & $32.2 \pm 7.7$ & $23.4 \pm 10.2$ & $14.7 \pm 7.7$ \\
\hline Number of medicinal uses & $17.6 \pm 3.1$ & $14.4 \pm 3.2$ & $16.7 \pm 1.9$ & $12.6 \pm 3.3$ & $12.8 \pm 4.6$ \\
\hline
\end{tabular}

${ }^{1)}$ Times per month; ${ }^{2)}$ Number of harvest holes per collection event; ${ }^{3)}$ US $\$=2422$ Ariary, 9.07.2014. 
by the informants. Knowledge, traditional uses and the number of species used differ significantly $(\mathrm{P}<0.01)$ among villages. Overall, the knowledge and the uses of plants are higher in Ampotake than in the other villages. In Ampotake, Miarintsoa and Itomboina, similar medicinal plant species are used as indicated by the Jaccard similarity indices ranging between 0.68-0.7 (Table 7).

\section{Effects of socio-economic characteristics on the use and knowledge of plants}

The number of livestock owned (TLU), education level, family size and agricultural harvest were significant predictors for the number of medicinal plants used and the frequency of yam collection. The TLU and the age of respondents significantly affected the collection of wild yams $(\mathrm{P}<0.001$; Table 8$)$. In the study region, a high number of livestock owned is a sign of wealth. Households with a low TLU are characterized by higher yam collection intensities. For the number of medicinal plants used, the only significant predictor variables were family size and healer consultancy. The latter indicates how often a household asked a traditional healer for advice on appropriate medicinal plants. The higher the diversity of different household activities (salaried work, trading, artisanal), the more cash income is produced. Consequently, the households have the possibility to buy food during difficult seasons, and depend less on wild food collection. In addition, female respondents use and know more plants than men. Age did not affect the use and knowledge on medicinal plants, which is maybe due to the direct knowledge transfer within one household. In this study, $79 \%$ of the households did not report to consult a traditional healer in case of illness.

\section{Discussion}

\section{Characteristics of the interviewed households}

The basic characteristics of the interviewed households correspond to the results of INSTAT [22] for SW Madagascar even though our survey indicated a higher education level. In Ampotake, the majority of the households heads (52\%) are illiterate, which reflects the

Table 7 Similarity among medicinal plant species usage in the studied villages (Jaccard similarity indices, 1 = similar) in the Mahafaly region of SW Madagascar

\begin{tabular}{llllll}
\hline & Ampotake & Andremba & Itomboina & Miarintsoa & Efoetse \\
\hline Ampotake & 1 & 0.59 & 0.7 & 0.68 & 0.54 \\
Andremba & 0.59 & 1 & 0.58 & 0.58 & 0.43 \\
Itomboina & 0.7 & 0.58 & 1 & 0.71 & 0.55 \\
Miarintsoa & 0.68 & 0.58 & 0.71 & 1 & 0.51 \\
Efoetse & 0.54 & 0.43 & 0.55 & 0.51 & 1 \\
\hline
\end{tabular}

percentage of the non-educated people in the rural area in this region. The average land size per household (2.2 ha) corresponds to the respective value in Mozambique [40]. In this study, we used off-farm activities to determine the different cash income sources and diversification level of households based on the assumption that higher diversification leads to higher income $[41,42]$.

\section{Traditional knowledge and usage of wild yams}

Among the six species of wild yam recorded, only $D$. alatipes and $D$. bemandry were frequently harvested by local people to substitute for staple food. This is comparable to the collection of wild yam species in the dry forest of NW-Madagascar [43]. Mavengahama et al. [44] recorded a similar importance of wild yam collection for rural livelihoods in South Africa, where wild vegetable are of high importance in supplementing staple food diets based on maize, sorghum (Sorghum bicolor Moench.), and millet (Pennisetum glaucum L.).

In our study, the collection intensity of wild yams depended not only on the availability of the species, but also on the taste of the yam tubers. For Malagasy yams, the preference in taste was analysed by Jeannoda et al. [14] who observed a significant correlation $(\mathrm{P}<0.001)$ between the preference and the sensitivity to saccharose. Polycarp et al. [45] stated that the high level of carbohydrate and energy with appreciable levels of minerals makes yam a very nutritious source of food. Bhandari et al. [46] found that the nutritional composition of selected wild yams in Nepal was similar to those reported for cultivated species of yam. When analyzing the nutritional value of Malagasy yam germplasm, including those of wild species, Jeannoda et al. [14] determined high contents of calcium in Dioscorea ovinala, which makes some wild yams physiologically important.

However, a decline in the availability of wild yams was already reported by the respondents of our study who are forced to increase the search radius for tuber harvests. One main reason for the decline in this essential resource securing local livelihood strategies against drought related hunger risks may be the exploitative harvesting methods used by the majority of the collectors in the Mahafaly region, which hampers the regeneration of the species. In contrast, Ackermann [43], who conducted a study in the NW-Madagascar reported that traditional people try to harvest the tubers carefully to guarantee the survival of the plant stand. In our study only $15 \%$ of the household took care of the regeneration of the lianas. While the sale of wild yam tubers provides valuable cash income for many households it may also be one of the causes for its overexploitation and increasingly threatened existence [47]. About 20\% of the harvested tubers per households are sold on local markets. 
Table 8 Generalized linear Model (GLM) showing the effect of selected independent variables on the number of medicinal plants used and the collection frequency of wild yam $(n=250)$ in rural villages of the Mahafaly region in SW-Madagascar

\begin{tabular}{|c|c|c|c|c|c|c|}
\hline \multirow[t]{2}{*}{ Independent variable } & \multicolumn{3}{|c|}{ Number of medicinal plants used } & \multicolumn{3}{|c|}{ Frequency of yam collection (Frequency month- ${ }^{-1}$ ) } \\
\hline & $B^{*}$ & $\mathbf{P}$ & $r$ & B & $\mathbf{P}$ & $\mathbf{R}$ \\
\hline Education level & -0.087 & .029 & -0.083 & -0.249 & 0.008 & -0.118 \\
\hline Tropical livestock unit & -0.007 & .038 & -0.192 & -0.460 & 0.000 & -0.263 \\
\hline Agricultural harvest & -0.127 & .002 & -0.270 & -0.251 & 0.012 & -0.229 \\
\hline Age & 0.002 & .217 & 0.119 & -0.014 & 0.000 & -0.209 \\
\hline Family size & 0.027 & .001 & 0.119 & 0.056 & 0.003 & 0.092 \\
\hline Gender & 0.125 & .029 & 0.128 & 0.153 & 0.232 & 0.124 \\
\hline Healer consultancy & -0.472 & .000 & -0.380 & - & - & - \\
\hline Households activities & - & - & - & 0.053 & 0.550 & 0.038 \\
\hline
\end{tabular}

(*) Beta coefficient; $(r)$ regression coefficient, $(-)$ the variable was not included in the model.

Traditional knowledge and usage of medicinal plants

The majority of the medicinal plants used by the local people belong to the Fabaceae, Apocynaceae and Euphorbiaceae. In contrast to yams, none of the interviewed households was selling medicinal plants. Local people complained that some species are nowadays hard to find, which was confirmed by our field observation. Hamilton [48] stated that globally 4,160 to 10,000 medicinal plants are endangered by habitat losses or overexploitation in areas where rural families traditionally collected them. The present study shows that the most popular plants with high use values, such as Aloe divaricata, Erythroxylum retusum, Cedrelopsis grevei, Neobeguea mahafaliensis, Salvadora angustifolia and Croton geayi are native species collected from forest habitats. This shows that the wild habitats are important for local communities in terms of basic needs. Beltrán-Rodríguez et al. [49] also pointed to the importance of wild habitats for peoples' livelihood in a rural community of Mexico and found a greater diversity of plant uses in wild habitats than in managed environments.

Some plants are less frequently used, which does not decrease their importance since most of them are needed for very specific therapeutic purposes. The increasing scarcity of such plants may also enhance the loss of traditional knowledge about the medicinal uses $[50,51]$. On the other hand there are cultivated species such as Tamarindus indica and Sclerocarya birrea subsp. caffra, Citrullus lanatus and Ziziphus spinachristi, which are nowadays used more intensively for medicinal purposes.

Different parts of the same plant are used for different purposes or by different population groups. Sometimes, a specific plant part is used for children and another part of the same plant for adults to treat a disease such as in the case of Aloe divaricate. The use of plant roots as traditional remedies is often problematic as it prevents plant regeneration [52]. Muthu et al. [53] reported that the choice of plant species most used by people depended largely on the type of diseases treated. In our study, digestive disorders, post-delivery care, body injuries and wounds were the most frequently mentioned diseases. This is comparable to similar studies conducted in Africa [54,55] China [56] and in Colombia [57], where digestive disorders were most frequently treated by medicinal plants. Compared to other developing countries, where sexually transmitted infections are most commonly treated with herbal medicines [58] this category was rarely cited in our study. Except for venereal diseases which are treated using a combination of different species $[59,60]$ the majority of plant species utilized had a single therapeutic use.

Some of the recorded medicinal plants in Madagascar are already pharmaceutically analysed and the active ingredients confirm traditional therapeutic uses. For example, Koehneria madagascariensis has a large and strong antimicrobial activity [61]. Hernandia voyronii [62] is known for its antimalarial active substances, Neobeguea mahafaliensis and Cedrelopsis greveii for effectiveness against cardiovascular diseases [63]. Although the World Health Organization (WHO) reported that 60$70 \%$ of Madagascar inhabitants have ready access to primary health care [64], accessibility of effective modern medicines is still a challenge for the local population in the Mahafaly region and they thus make use of native plants for alternative treatment.

\section{Effects of socio-economic conditions on the use of wild yams and medicinal plants}

Our study revealed that the collected quantities and qualities of plants vary greatly between households. Very poor and poor farmers consume and sale more yams and have higher knowledge on traditional usages of medicinal plants than well-off or "rich" individuals. Households with lacking off-farm income collect and consume more frequently wild yams than households with regular off- 
farm income. In addition, the regression results revealed, that households with more cropland and higher crop harvest collect less forest products. This was also confirmed by Reddy and Chakravarty [65] in India. Variables showing the collection and consumption of wild yams $(\mathrm{P}<0.01)$ were important discriminators for household groups in contrast to the variables on the use of medicinal plants $(\mathrm{P}<0.05)$.

The use of forest products was significantly higher in villages near forests, where wild yams and medicinal plants are more readily available. This confirms findings of Banana and Turiho-Habwe [66] in Uganda and Kerapeletswe and Lovett [67] in Botswana, where the dependency on the forests for food supply decreased rapidly with an increasing distance of the respondent's home from the forests. Furthermore, poor market access may increase the importance of forest products to sustain people's livelihood [68].

The number of livestock owned by the household, education level, agricultural harvest and family size affected the collection of wild yams and the usage of medicinal plants. Livestock and off farm activities determine the wealth condition of the household in this region and were negatively correlated with the use of wild yams and medicinal plants. However, we cannot generalize these findings as with time and location the direction of the relationship may change [69]. Socio-cultural factors are of higher importance for the use of medicinal plants than for the collection of wild yams. In contrast to other findings [49] female respondents use more plant species than males. The use of medicinal plants is the basic health care for the majority of the households and the knowledge about their use was maybe shared over generations, which might explain, that there is no significant influence of informant age on the collection intensity of medicinal plants. In the study of Kirstin [70] on the usage of Budongo's forest products, the use of wild food such as Dioscorea spp. increased with age, whereas young village people focused on the use of fruits and wild game because of their higher income potential. This might also be true for our study region, were younger farmers predominate in collecting wild yams for sale.

Overall, this study indicates that a household's wealth status affects the traditional knowledge and use intensity of forest products, which confirms previous studies $[49,71,72]$. The World Resources Institute [4] reported that families facing poverty, sickness, drought, wars and economic crisis depend to a higher degree on the collection of wild resources. Although, our study focused only on medicinal plants and wild yams as forest products, the rate of change in social and economic attributes of rural households is likely proportional to the rate of change in resource use [73]. Therefore, whatsoever the products extracted, a household's socio-economic dynamics ultimately drives its ability to use village forest resources.

\section{Conclusions}

Our results revealed that wild yams play an important role in local food security in the Mahafaly region, especially for poor farmers. On the other hand, medicinal plants are a primary source of health care for the majority of local people in SW-Madagascar and the results of this study can help to identify the most useful plant species and their importance for the local people. In many rural areas of developing countries, common property resource management plans may allow to combine poverty reduction and biodiversity conservation. In our study region the forest patches around the Tsimanampetsotsa National Park are managed by local communities. Our results indicate the influence of socioeconomic household characteristics on the use of forest products and its intensity, which should be considered in future management plans for local and regional forest conservation.

\section{Competing interests}

The authors declare that they have no competing interests.

\section{Authors' contributions}

JNA conducted the data collection, carried out the analyses and the interpretation of the results and wrote a first draft of the manuscript. KB supervised the field research and statistical analysis, contributed to interpretation of the results and writing of the final manuscript. $A B$ and $V J$ designed the research project, contributed with original ideas and reviewed the manuscript. All authors read and approved the final manuscript.

\section{Acknowledgements}

We would like to thank the local people of the Mahafaly region for their trust and hospitality during the field part of this study. We also acknowledge the SuLaMa project team, especially the group of interviewers supervised by Regina Neudert and Miandrazo Rakotoarisoa, for their support during data collection. We thankfully acknowledge the support of DAAD for the scholarship grant for this research and the German Federal Ministry of Education and Research for funding (BMBF, FKZ: 01LL0914C).

\section{Author details}

'Organic Plant Production and Agroecosystems Research in the Tropics and Subtropics, University of Kassel, Witzenhausen, Germany. 'Department of Biology and Vegetation Ecology, University of Antananarivo, Antananarivo, Madagascar.

Received: 25 July 2014 Accepted: 24 November 2014

Published: 30 December 2014

\section{References}

1. Myers N, Mittermeier RA, Mittermeier CG, da Fonseca GAB, Kents J: Biodiversity hotspots for conservation priorities. Nature 2000, 403:853-858.

2. UNPF (United Nations Population Fund: State of World Population. New York, USA: Reaching Common Ground: Culture, Gender and Human Rights; 2008.

3. UNDP (United Nations Development Programme): Human Development Report. New York: The Rise of the South: Human Progress in a Diverse World. United Nations development Programme; 2013.

4. WRI (World Resources Institute): The Wealth of the poor: Managing ecosystems to fight poverty. Washington, DC: 2005. www.iied.org.

5. Olson DM, Dinerstein E: The global 200: Priority Ecoregions for Global. Ann Mo Bot Gard 2002, 89:199-224.

6. Bationo A, Lompo F, Koala S: Research on nutrient flows and balances in West Africa: State-of-the-art. In Nutrient balances as indicators of production and sustainability in sub-Saharan African agriculture, Agriculture, Ecosystems and Environment, Volume 71. Edited by Smaling EMA; 1998:19-36. 
7. Buerkert A, Hiernaux P: Nutrients in the West African Sudano-Sahelian zone: losses, transfers and role of external inputs. J Plant Nutr Soil Sci 1998, 161:365-383

8. SuLaMa (Sustainable Landmanagement in south-western Madagascar: Recherche participative pour appuyer la gestion durable des terres du Plateau Mahafaly dans le sud-ouest de Madagascar. In Diagnostic participatif de la gestion des ressources naturelles sur le plateau Mahafaly Commune Rurale de Beheloka, Toliara. Madagascar: Rapport Final. Project SuLaMa; 2011.

9. The SuLaMa: (Sustainable Landmanagement in south-western Madagascar) webpage. [http://www.sulama.de/index.php/en/]

10. Shippmann U, Leaman DJ, Cunningham AB: Impact of Cultivation and Gathering of Medicinal Plants on Biodiversity: Global Trends and Issues Published in Biodiversity and the Ecosystem Approach in Agriculture, Forestry and Fisheries. Satellite event on the occasion of the Ninth Regular Session of the Commission on Genetic Resources for Food and Agriculture. Rome: Inter-Departmental Working Group on Biological Diversity for Food and Agriculture; 2002.

11. Cao S, Kingston DGl: A biodiversity conservation and drug discovery: can they be combined? The suriname and Madagascar Experiences. Pharm Biol 2009, 47:809-823.

12. Rasoanaivo P: Traditional medicine Programs in Madagascar. Washington, DC: World Bank; 2006. https://openknowledge.worldbank.org/handle/10986/ 10739.

13. Burkill $\mathrm{H}$, Perrier de la Bathie $\mathrm{H}$ : Dioscoreaceae dans la flore de Madagascar et des Comores. Paris: Museum d'Histoire Naturelle; 1950.

14. Jeannoda VH, Razanamparany JL, Rajaonah MT, Monneuse MO, Hladik A, Haladik CM: Les ignames (Dioscorea spp.) de Madagascar: espèces endémiques et formes introduites; diversité, perception, valeur nutritionnelle et systèmes de gestion durable. Revue d'Ecologie Terre Vie 2007, 62:191-207.

15. Tostain S, Cheban AS, Damson S, Mananjo H, Rejo-Fienena F: Les espèces d'ignames (Dioscorea sp.) dans le Sud de Madagascar, inventaires et aires de répartition. In Les ignames malgaches, une ressource à préserver et à valoriser. Actes du colloque. Edited by Tostain S, Rejo-Fienena F. Toliara: University of Toliara; 2010:22-39.

16. Bardhan P, Dayton-Johnson J: Heterogeneity and Commons Management Bloomington, Indiana: Proceedings of the 8th International Conference on Common Property. IASCO; 2000.

17. Elmqvist $T$, Pyykönen $M$, Tengö $M$, Rakotondrasoa F, Rabakonandrianina $E$, Radimilahy C: Patterns of loss and regeneration of tropical dry forest in Madagascar: the social institutional context. PLoS One 2007, 2(5):e402. doi:10.1371/journal.pone.0000402

18. Du Puy DJ, Moat JF: Vegetation mapping and classification in Madagascar (using GIS): implications and recommendations for the conservation of biodiversity. In Chorology, taxonomy and ecology of the African and Madagascan floras. Edited by Huxley CR, Lock JM, Cutler DF. Kew: Royal Botanic Gardens; 1998:97-117.

19. UPDR (Unité de Politique pour le Développement Rural): Monographie de la Région Sud-Ouest. Madagascar: Ministere de l'agriculture, de l'élevage et de la pêche; 2003

20. Sussman RW, Green GM, Porton I, Andrianasolondaibe OL, Ratsirarson JA Survey of the habitat of Lemur catta in southwestern and southern Madagascar. Primate Conserv 2003, 19:32-57.

21. Brinkmann K, Fanambinantsoa N, Ratovonamana RY, Buerkert A: Causes of deforestation processes in southwestern Madagascar during the past 40 years: What can we learn from settlement characteristics? Agric Ecosyst Environ 2014, 195:231-243

22. Institut National de la Statistique (INSTAT): Enquête Périodique auprès des Ménages. Antananarivo: République de Madagascar; 2010.

23. WFP (World Food Programme): Global food security update. Washington, DC Tracking food security trends in vulnerable countries; 2013.

24. Mamokatra: Etude pour l'élaboration d'un plan d'amén-agement et de gestion au niveau de la Reserve Naturelle Intégrale de Tsimanampetsotsa: Diagnostic physic-bio-écologique. Deutsche Forstservice GmbH. Antananarivo: Feldkirchen et Entreprise d'Etudes de Développement rural; 1999.

25. Ratovomanana RY, Rajeriarison C, Roger E, Kiefer I, Ganzhorn JU: Impact of livestock grazing on forest structure, plant species composition and biomass in southwestern Madagascar. In Scripta Botanica Belgicaheld, Volume 50. Antannanarivo, Madagascar: African Plant diversity, Systematic and Sustanaible Development-Proceedings of XIXth AETFAT congress; 2013:82-92.
26. Neudert R, Andriamparany NJ, Rakotoarisoa M, Götter J: Income diversification, wealth, education and well-being in rural south-western Madagascar: Results from the Mahafaly Region. Dev South Afr. in press.

27. Stepp JR: Advances in ethnobiological field methods. Field Methods 2005, 17:211-218.

28. Berlin EA: Some field methods in medical ethnobiology. Field Methods 2005, 17:235-268.

29. Martin G: Ethnobotany: A methods manual. London, UK: Chapman \& Hall; 1995.

30. Tropicos.org. Missouri Botanical Garden. http://www.tropicos.org.

31. Cook FEM: Economic botany data collection standard. Kent, UK: Royal Botanic Gardens; 1995

32. Simpson EH: Measurement of diversity. Nature 1949, 163:688.

33. Byg A, Balslev H: Diversity and use of palms in Zahamena, eastern Madagascar. Biodivers Conserv 2001, 10:951-970.

34. Phillips O, Gentry AH: The useful plants of Tambopata, Peru. Statistical hypotheses tests with a new quantitative technique. Econ Bot 1993, 47:15-32.

35. Albuquerque UP, Medeiros PM, Almeida AL, Monteiro JM, LinsNeto EMF, Melo JG, Santos JP: Medicinal plants of the caatinga semi-arid vegetation of NE Brazil. A quantitative approach. J Ethnopharmacol 2007, 114:325-354.

36. Mueller-Dombois D, Ellenberg $\mathrm{H}$ : Aims and methods of vegetation ecology. New York: John Wiley \& Sons; 1974

37. Burnham KP, Anderson DR: Model Selection and Multimodel Inference: a practical information-theoretic approach. 2nd edition. New York: Springer-Verlag; 2002. 0-387-95364-7.

38. Food and Agriculture Organization (FAO): Production Yearbook 1985, Volume 39. Rome: FAO; 1986

39. Ali S: Research methodology: Data Analysis. ABAC J 1999, 19:52-74

40. Jayne TS, Yamano T, Weber M, Tschirley D, Benfica R, Chapoto A, Zulu B: Smallholder income and land distribution in Africa: Implications for poverty reduction strategies. Food Policy 2003, 28:253-275.

41. Beyene AD: Determinants of off-farm participation decision of farm households in Ethiopia. Agrekon 2008, 47:140-161.

42. Bigsten A, Tengstam S: Smallholder diversification and income growth in Zambia. J Afr Econ 2011, 20:781-822.

43. Ackermann K: Utilisation of wild growing yams as supplementary nutrition and its impact on the dry forest ecosystem in north-western Madagascar. Schweizerische Zeitschrift Für Forstwesen 2004, 155:80-88.

44. Mavengahama S, McLachlan M, Clercq W: The role of wild vegetable species in household food security in maize based subsistence cropping systems. Food Secur 2013, 5:227-233.

45. Polycarp D, Afoakwa EO, Budu AS, Otoo E: Characterization of chemical composition and anti-nutritional factors in seven species within the Ghanaian yam (Dioscorea) germplasm. Intern Food Res J 2012, 19:985-992.

46. Bhandari MR, Kasai T, Kawabata J: Nutritional evaluation of wild yam (Dioscorea spp.) tubers of Nepal. Food Chem 2003, 82:619-623.

47. Termote C, Damme P, Dhed'a Djailo B: Eating from the wild: Turumbu, Mbole and Bali traditional knowledge on non-cultivated edible plants, District Tshopo, DRCongo. Genet Resour Crop Evol 2010, 58:585-618.

48. Hamilton AC: Medicinal plants, conservation and livelihoods. Biodivers Conserv 2004, 13:1477-1517.

49. Beltrán-Rodríguez L, Ortiz-Sánchez A, Mariano NA, Maldonado-Almanza B, Reyes-García V: Factors affecting ethnobotanical knowledge in a mestizo community of the Sierra de Huautla Biosphere Reserve Mexico. J Ethnobiol Ethnomed 2014, 10:14

50. Benz BF, Santana F, Pineda R, Cevallos J, Robles L, DeNiz D: Characterization of mestizo plant use in the Sierra de Manantlán, Jalisco-Colima, México. J Ethnobiol 1994, 14:23-41.

51. Chaudhary MI, He Q, Cheng YY, Xiao PG: Ethnobotany of medicinal plants from Tian Mu Shan Biosphere Reserve, Zhejiang-Province, China. Asian J Plant Sci 2006, 5:646-653

52. Flatie T, Gedif T, Asres K, Gebre-Mariam T: Ethnomedical survey of Berta ethnic group Assosa Zone, Benishangul-Gumuz regional state, mid-west Ethiopia. J Ethnobiol Ethnomed 2009, 5:14. doi:10.1186/1746-4269-5-14 
53. Muthu C, Ayyanar M, Raja N, Ignacimuthu S: Medicinal plants used by traditional healers in Kancheepuram district of Tamil Nadu. India J Ethnobiol Ethnomed 2006, 2:43. doi:10.1186/1746-4269-2-43.

54. Maroyi A: Traditional use of medicinal plants in south-central Zimbabwe: review and perspectives. J Ethnobio/ Ethnomed 2013, 9:31. doi:10.1186/ 1746-4269-9-31.

55. Ribeiro A, Romeiras MM, Tavares J, Faria MT: Ethnobotanical survey in Canhane village, district of Massingir, Mozambique: medicinal plants and traditional knowledge. J Ethnobiol Ethnomed 2010, 6:33. doi:10.1186/1746-4269-6-33.

56. Ghorbani A, Langenberger G, Feng L, Sauerborn J: Ethnobotanical study of medicinal plants utilised by Hani ethnicity in Naban River Watershed National Nature Reserve, Yunnan, China. J Ethnopharmacol 2011 134:651-667.

57. Cadena-González AL, Sørensen M, Theilade I: Use and valuation of native and introduced medicinal plant species in Campo Hermoso and Zetaquira, Boyacá, Colombia. J Ethnobiol Ethnomed 2013, 9:23. doi:10.1186/1746-4269-9-23

58. Van Vuuren SF, Naidoo D: An antimicrobial investigation of plants used traditionally in southern Africa to treat sexually transmitted infections. J Ethnopharmacol 2010, 130:552-558.

59. Zonyane S, Van Vuuren SF, Makunga NP: Pharmacological and phyto-chemical analysis of a medicinal plant mixture that is used as traditional medicine in Western Cape. In Paper presented at South Africa Association of Botanist 38th Annual Conference (15-18 January 2012). Pretoria: University of Pretoria; 2012.

60. Bussmann RW, Sharon D: Traditional medicinal plant use in northern Peru: tracking two thousand years of healing culture. J Ethnobiol Ethnomed 2006, 2:47. doi:10.1186/1746-4269-2-47.

61. Rakotonirina EF, Rajaonarison JF, Raoelison EG, Rajaonarivelo JP, Manga N, Solofoniaina M, Rakotonirina B, Randriamampionona D, Rabemanantsoa C, Cheuk K, Urveg-Ratsimamanga S, Leclercq JQ: Antimicrobial activity of 23 endemic plants in Madagascar. Trop J Pharm Res 2010, 9:165-171.

62. Ratsimamanga-Urverg S, Rasoanaivo P, Milijaonina R, Rakotoarimanga J, Rafatro H, Robijaona B, Rakoto-Ratsimamanga A: In votro antimalarial activity, chlorochuine potentiating effect and cytotoxicity of alkaloids of Hernandia voyroni. Phytoterapy Res 1994, 8:18-21.

63. Ralay Ranaivo H, Rakotoarison O, Tesse A, Schott C, Randriantsoa A, Lobstein $A$, Andriantsitohaina R: Cedrelopsis grevei induced hypotension and improved endothelial vasodilatation through an increase of $\mathrm{Cu} / \mathrm{Zn}$ SOD protein expression. Am J Physiol Heart Circulatory Physiol 2004, 286:775-781.

64. WHO (World Health Organization): Primary health care: back to basics in Madagascar. Bull World Health Org 2008, 86:417-496.

65. Reddy SRC, Chakravarty SP: Forest dependence and income distribution in a subsistence economy: Evidence from India. World Dev 1999, 27:1141-1149.

66. Banana AY, Turiho-Habwe W: A socio-economic analysis of forest foods consumption: the local community use of forests of Hoima and Masindi districts, western Uganda. African Crop Sci 1997, 3:1435-1442.

67. Kerapeletswe CK, Lovett JC: The likely effects of inequality and globalization on sustainable management of common pool resources: The case of Basarwa (Bushmen) of Botswana. http://dlc.dlib.indiana.edu/ dlc/handle/10535/1173.

68. Gunatilake $\mathrm{H}$ : The role of rural development in protecting tropical rainforests: evidence from Sri Lanka. J Environ Manag 1998, 53:273-292.

69. Mcelwee PD: Forest environmental income in Vietnam: household socioeconomic factors influencing forest use. Environ Conserv 2008, 35:147-159.

70. Kirstin RJ: Local use of Budongo's forest products. In M.Sc. Thesis. U.K University of Oxford; 1993.
71. Khanal KP: Economic evaluation of community forestry in Nepal and its equity distribution effect. In M.Sc. Thesis. Copenhagen, Denmark: The Royal Veterinary and Agricultural University; 2001.

72. Sapkota IP, Odén PC: Household characterstics and dependency on community forests. Int J Soc Forestry 2008, 1:123-144.

73. Kant S: A dynamic approach to forest regimes in developing economies. Ecol Econ 2000, 32:287-300.

doi:10.1186/1746-4269-10-82

Cite this article as: Andriamparany et al: Effects of socio-economic household characteristics on traditional knowledge and usage of wild yams and medicinal plants in the Mahafaly region of south-western Madagascar. Journal of Ethnobiology and Ethnomedicine 2014 10:82.

\section{Submit your next manuscript to BioMed Central and take full advantage of:}

- Convenient online submission

- Thorough peer review

- No space constraints or color figure charges

- Immediate publication on acceptance

- Inclusion in PubMed, CAS, Scopus and Google Scholar

- Research which is freely available for redistribution
C Biomed Central 